\title{
LES/PDF modeling of autoignition in a lifted turbulent flame: Analysis of flame sensitivity to differential diffusion and scalar mixing time-scale
}

\author{
Wang Han ${ }^{\mathrm{a}, \mathrm{b}, *}$, Venkat Raman ${ }^{\mathrm{a}}$, Zheng Chen $^{\mathrm{b}}$ \\ ${ }^{a}$ Department of Aerospace Engineering, The University of Michigan, Ann Arbor, MI 48109, USA \\ ${ }^{b}$ SKLTCS, Department of Mechanics and Engineering Science, College of Engineering, Peking University, \\ Beijing 100871, China
}

\begin{abstract}
The stabilization of lifted flames involves a complex competition between small-scale mixing and propagation of flame kernels at the base of the flame. Here, the effect of species diffusivity on this stabilization process is explored. For this purpose, a large-eddy simulation (LES)/probability density function (PDF) methodology accounting for differential diffusion is developed. A dynamic model for scalar mixing time-scale is formulated to accurately describe the turbulence-driven mixing of scalars at the small-scales. Autoignition-stabilized $\mathrm{H}_{2} / \mathrm{N}_{2}$ lifted turbulent flames in a vitiated coflow burner are chosen as test cases. Detailed chemical kinetics is used along with in situ adaptive tabulation (ISAT) to accelerate the computations. Four configuration test cases are considered: neglecting differential diffusion (Cases 1 and 2), considering differential diffusion (Case 3) and considering differential diffusion but neglecting molecular transport of $\mathrm{H}$ and $\mathrm{H}_{2}$ (Case 4). A dynamic model for scalar mixing time-scale appearing in the mixing model is used in Cases 2, 3 and 4, which removes the need to specify the value of model constant. It is found that the lifted flames are very sensitive to differential diffusion. The predictions of major species mass fractions, temperatures and lift-off heights are in good agreement with the experimental data. Further, such agreement could be achieved without changing any boundary conditions. In particular, increased diffusion of species upstream allows ignition kernels to survive turbulent dissipation, causing stabilization to occur upstream. $\mathrm{H}_{2}$ and $\mathrm{H}$ are identified as two critical species that play a dominant role in the differential diffusion effect. The model is also able to capture the change in lift-off height with change in coflow temperature. The results show that even in turbulent flames, stabilization of the flame zone can be sensitive to molecular diffusion. In LES computations where a significant part of this diffusion effect is resolved, the use of the enhanced LES/PDF/ISAT approach is seen as a promising approach for capturing the lift-off physics accurately.
\end{abstract}

Keywords: LES/PDF/ISAT simulation, autoignition, lifted flame, differential diffusion, scalar mixing time-scale 


\section{Introduction}

Turbulent jet flames in practical burners may be lifted from the base in order to protect the burner material [1]. Additionally, such lifted flames allow higher operational velocities that will increase energy throughput of the system. The stabilization, liftoff, and blowoff of these flames are dictated by the complex interaction between turbulence and chemical kinetics. Understanding these interactions is crucial for determining the operability regime as well as the emissions from the burner. In the past, a number of studies [1-4] have been devoted to the characterization of these physics. In addition, both computational [5-9] and experimental [10-12] studies have been performed to identify the flame stabilization mechanism and analyze the sensitivity of the lift-off height to jet velocity and coflow temperature. From these studies, two stabilization mechanisms have been identified: autoignition and premixed flame propagation. If the flame is stabilized by autoignition, a convection-diffusion-reaction balance is present at the flame base. On the other hand, when the flame is stabilized by premixed flame propagation, a convection-diffusion balance exists upstream of the flame base followed by a diffusion-reaction balance at the reaction zone. In practical flames, the two mechanisms often coexist. In this work, the focus is on autoignition-dominated flames.

Autoignition-stabilized lifted flames are particularly sensitive to radical formation and small-scale mixing [1-3]. In particular, two key parameters, namely the ignition delay time and the mixing time play an important role in determining the lift-off height. From a modeling viewpoint, this implies that both the chemical kinetics of flame stabilization and turbulent as well as molecular mixing of species need to be modeled accurately. Another important manifestation of such lifted flames is the presence of a partially-premixed flame base associated with low-probability events, such as extinction and re-ignition. Consequently, the turbulence-thickened flame base poses a multitude of modeling challenges. The standard non-premixed flamelet methods $[1,3,13]$ may not be adequate around the flame base. Recently, unsteady flamelet/progress variable (UFPV) models with a presumed subfilter probability density function (PDF) for mixture fraction and progress variable have been applied for this purpose [14, 15]. Here, unity Lewis numbers are assumed for species diffusive-transport in order to simplify the flamelet equations. An alternative approach is based on the transported-PDF formulation, which has been shown to be accurate for a range of turbulent flame problems [16-21]. This approach has also successfully predicted autoignition in lifted flames [22-28]. However, molecular diffusion is almost always handled using a single or mixture-averaged diffusivity in these studies. The focus here is to understand the role of species-related diffusivity on flame stabilization.

Molecular transport of species through diffusion, especially when multiple species with varying diffusivities are present, is a topic of current interest $[21,29,30]$. In this context, the flamelet models and PDF methods (in general) have differing approaches to handling species diffusivities. In the flamelet-approach, species-specific diffusion is incorporated at the level of the flamelet structure. This implies that such molecular effects are accounted

\footnotetext{
${ }^{*}$ Corresponding author

Email address: whanum@umich.edu (Wang Han)
} 
for in the solution to the one-dimensional flamelet equations. On the other hand, the spatial transport of this flame structure is through a conserved scalar (mixture fraction) and a reactive scalar representation. These scalars are necessarily transported using a single diffusivity. Consequently, the effect of differential diffusion at scales larger than the flame scales are neglected (or at best, inaccurately modeled). Blanquart et al. [31] have sought to address this issue by constructing mixture fraction equations that contain source terms to account for the change in species balance arising from differential diffusion. However, application of this approach to detailed chemistry based models is still being pursued. In the context of PDF methods, differential diffusion manifests in two different ways again. At the resolved scales, the PDF is altered by molecular diffusion through a second-order diffusion term in the PDF transport equation $[32,33]$. At the small-scales, the conditional diffusion term needs to incorporate the species-specific diffusivities in order to describe the small-scale mixing effect $[29,33]$. Since the conditional diffusion term involves two-point statistics, it needs to be exclusively modeled through an appropriate mixing model. Although there have been some advances in this context [29,33], the issue of mixing models is still an open question.

From a computational perspective, the relative importance of small-scale and resolvedscale differential diffusion depends on the turbulence modeling framework as well. In the Reynolds-averaged Navier-Stokes (RANS) approach, almost all of the turbulence energy cascade is modeled. In other words, the accuracy of the sub-grid models are extremely critical in capturing the flow physics. In gradient-diffusion hypothesis based closures for the Reynolds stress and turbulent scalar fluxes, the turbulent viscosity/diffusivity is considerably higher than the molecular component. Consequently, sub-grid description of differential diffusion becomes more critical than the resolved or large scale molecular transport of species. In this sense, the flamelet-type small-scale description is a better approach for modeling differential diffusion. Although such physics have been incorporated into conditional-diffusion models for the PDF approach as well [33], their accuracy has not been widely tested.

On the other hand, resolved molecular transport can be the same order of magnitude as the sub-filter turbulent transport in the LES context [21, 34]. This is mainly due to the fact that LES resolves large scale transport directly, and sub-filter turbulent diffusivity only captures the small-scale turbulence (in this paper, "turbulent viscosity" and "turbulent diffusivity" denote the viscosity and diffusivity used to model the turbulent motions unresolved by the LES grid; "molecular diffusivity" denotes the diffusivity resolved by the LES grid). When the LES computational grid is well-refined, especially in regions close to the flame front, molecular diffusivity can become comparable in magnitude to turbulent diffusivity. Previous studies on turbulent jet flames have indicated that differential diffusion effect can not be negligible [35] when molecular and turbulent diffusivities are comparable, and it is necessary to include differential diffusion effect accurately in the combustion models in the LES context $[29,36]$. It is expected that this large scale differential diffusion can exert significant influence on the small-scale scalar mixing which is critical for autoignition, extinction, and reignition $[2,37-41]$. At the same time, due to the increased resolution of the turbulence cascade, the small-scale differential mixing is expected to play a less significant role $[42,43]$. Recently, Wang and Kim [21] investigated the effect of resolved molecular transport on the 
PDF modeling of Sandia Flame E. It was found that local extinction can be captured by including molecular transport effect, even when the sub-filter mixing model was not explicitly altered to account for the evolution of the scalar covariances according to the differential diffusion process. Nevertheless, the effect of differential diffusion was neglected due to the assumption of equal molecular diffusivity in [21]. Yang et al. [27] performed a posteriori comparisons between LES/PDF computations accounting for molecular diffusivity effects and compared with results from direct numerical simulation (DNS) of a lifted ethylene jet flame [7]. They found that differential diffusion can have a significant effect on the lifted flames. More recently, Wang [44] developed a consistent flamelet model with differential diffusion for turbulent non-premixed flames. The dependence of differential diffusion on Reynolds number was carefully treated.

With this background, the objective of this work is to evaluate the relative effect of small-scale mixing and differential diffusion on flame stabilization with application to an experimental flame configuration. For this purpose, a consistent LES/PDF approach that incorporates molecular diffusivities of individual species is combined with a dynamic timescale model for sub-filter mixing. This approach is then used to simulate a series of turbulent jet flames that is known to stabilize through autoignition. From these simulations, the critical species that play a dominant role in stabilizing the flame are identified.

A canonical lifted turbulent flame, in a vitiated coflow burner developed by Cabra et al. [10], is selected as the validation test case. This flame has been extensively studied in the past. Two main conclusions from previous experimental and numerical studies[10, 15, 22$24,26,45-56]$ can be drawn: autoignition is the dominant stabilization mechanism in this flame, and the lift-off height is very sensitive to the coflow temperature. In this work, the detailed chemical kinetics is used along with the in-situ adaptive tabulation (ISAT) algorithm [57] to accelerate the chemistry calculations. To analyze the flame sensitivity to differential diffusion and scalar mixing time-scale, four different test cases are considered: neglecting differential diffusion (Cases 1 and 2), considering differential diffusion (Case 3) and considering differential diffusion but neglecting molecular transport of $\mathrm{H}$ and $\mathrm{H}_{2}$ (Case 4). A dynamic model for scalar mixing time-scale is used in Cases 2, 3 and 4, which removes the need to specify the value of model constant.

The paper is organized as follows. First, the augmented LES/PDF/ISAT approach is summarized in the next section. The experimental configuration of the vitiated coflow burner and the numerical setups are presented in Sections 3 and 4, respectively. In Section 5, computational results are discussed. Finally, the conclusions are given in Section 6.

\section{LES/PDF approach}

The hybrid method consists of two separate solvers: a variable density low-Mach number LES solver and a Lagrangian particle PDF solver. While the LES solver evolves the momentum, energy, and continuity equations, the Lagrangian solver obtains the one-time one-point joint PDF of the thermochemical composition. In this section, details of this hybrid solver, including the handling of the differential diffusion, scalar mixing model, and the two-way coupling algorithm are discussed. 


\subsection{Low-Mach number LES solver}

LES is based on a high band-pass filtering operation, which separates the flow field into the resolved large scales that are computed on an Eulerian grid and the small-scales that are modeled. For a variable density reacting flow, the Favre-filtered quantity of a scalar $\varphi$ is computed as

$$
\widetilde{\varphi}(\boldsymbol{x}, t)=\frac{1}{\bar{\rho}} \int \rho(\boldsymbol{y}, t) \varphi(\boldsymbol{y}, t) G(\boldsymbol{y}-\boldsymbol{x}) d \boldsymbol{y}
$$

where $t$ is time, $\boldsymbol{x}$ is the vector of spatial coordinates, $\rho$ is the density, and $\bar{\rho}$ is the filtered density. $G$ is a filter kernel which is typically assumed be a box filter defined by the computational grid $[32,58]$. The LES equations can be obtained by applying the filtering operation to the instantaneous governing equations that describe the conservation of mass and momentum:

$$
\begin{gathered}
\frac{\partial \bar{\rho}}{\partial t}+\frac{\partial \bar{\rho} \widetilde{u}_{j}}{\partial x_{j}}=0 \\
\frac{\partial \bar{\rho} \widetilde{u}_{i}}{\partial t}+\frac{\partial \bar{\rho} \widetilde{u}_{i} \widetilde{u}_{j}}{\partial x_{j}}=-\frac{\partial \bar{p}}{\partial x_{i}}+\frac{\partial \sigma_{i j}}{\partial x_{j}}+\frac{\partial \sigma_{i j}^{s g s}}{\partial x_{j}}
\end{gathered}
$$

where $\widetilde{u}_{j}$ is the Favre-filtered velocity component, and $\bar{p}$ is the filtered hydrodynamic pressure. $\sigma_{i j}$ is the filtered viscous stress tensor with

$$
\sigma_{i j}=2 \widetilde{\mu} \widetilde{S}_{i j}=\widetilde{\mu}\left(\frac{\partial \widetilde{u}_{i}}{\partial x_{j}}+\frac{\partial \widetilde{u}_{j}}{\partial x_{i}}-\frac{2}{3} \frac{\partial \widetilde{u}_{k}}{\partial x_{k}} \delta_{i j}\right)
$$

and $\sigma_{i j}^{s g s}=\bar{\rho} \widetilde{u}_{i} \widetilde{u}_{j}-\bar{\rho} \widetilde{u_{i} u_{j}}$ is the sub-filter stress tensor. This sub-filter component is modeled using a dynamic procedure based turbulent viscosity assumption [58, 59].

The flows of interest here fall in the low-Mach number regime but the density is variable due to the reaction-related heat release and boundary conditions. For such configurations, the use of a low-Mach approximation accelerates the computations [58, 60]. Here, the lowMach number fractional time-stepping algorithm is used [58]. The critical component in this approach is the pressure projection applied to enforce mass conservation. The solver is advanced in time using a semi-implicit method in the radial and azimuthal directions, which allows a larger time-step to be used. The turbulent viscosity is obtained using a dynamic Smagorinsky approach [59]. More details of the solver are provided in [60, 61].

\subsection{Lagrangian PDF approach}

In the Lagrangian PDF approach, the one-point one-time PDF of the gas-phase thermochemical composition is directly computed. This composition $\phi$ contains the $n_{s}$ species mass fractions and sensible enthalpy $h$. The $\operatorname{PDF} f(\boldsymbol{\psi} ; \boldsymbol{x}, t)$ is defined as a mass density function $[28,43,62-64]$ :

$$
\bar{\rho}=\int f(\boldsymbol{\psi} ; \boldsymbol{x}, t) d \boldsymbol{\psi}
$$

and the filtered moments of composition can be computed based on the PDF as follows

$$
\widetilde{\phi_{p}^{m} \phi_{q}^{n}}=\frac{1}{\bar{\rho}} \int \psi_{p}^{m} \psi_{q}^{n} f d \boldsymbol{\psi}
$$


where $\boldsymbol{\psi}, \phi_{p}$ and $\phi_{q}$ denote the composition sample space variable and components of $\boldsymbol{\phi}$, respectively.

The PDF transport equation can then be written as [43, 62-64]

$$
\frac{\partial f}{\partial t}+\nabla \cdot(\widetilde{\boldsymbol{u}} f)+\nabla \cdot \widetilde{\left(\widetilde{\boldsymbol{u}^{\prime} \mid \boldsymbol{\psi}} f\right)}=-\frac{\partial}{\partial \psi_{\alpha}}\left[\left(\frac{1}{\bar{\rho}} \overline{\nabla \cdot\left(\rho D_{(\alpha)} \nabla \phi_{\alpha}\right) \mid \boldsymbol{\psi}}+S_{\alpha}(\boldsymbol{\psi})\right) f\right]
$$

where $\widetilde{\boldsymbol{u}^{\prime} \mid \boldsymbol{\psi}}$ is the sub-filter velocity fluctuation conditioned on the scalar, $\overline{\nabla \cdot\left(\rho D_{(\alpha)} \nabla \phi_{\alpha}\right) \mid \boldsymbol{\psi}}$ is the conditional micromixing term, and $S_{\alpha}$ is the chemical reaction source term for thermochemical composition component $\alpha . D_{(\alpha)}$ is the corresponding molecular diffusivity of the species. It is noted that the impact of differential diffusion between species can be studied based on Eq. 7. The conditional velocity term on the left-hand-side of Eq. 7 is modeled using the gradient-diffusion hypothesis $[17,62]$

$$
\left.\nabla \cdot \widetilde{\left(\boldsymbol{u}^{\prime} \mid \boldsymbol{\psi}\right.} f\right)=-\nabla \cdot\left[\bar{\rho} D_{t} \nabla(f / \bar{\rho})\right]
$$

where $D_{t}$ is turbulent diffusivity that is evaluated using a dynamic approach similar to that used to obtain the turbulent viscosity [61].

The conditional mixing term needs to be modeled and has received considerable attention in the context of transported-PDF methods [17, 33, 41]. In spite of the substantial efforts to develop mixing models, a general model that is robust and that meets all modeling requirements is not currently available [32, 41]. Here, it is modeled using the Interactionby-Exchange-with-the-Mean (IEM) model [65]. The resolved part of differential diffusion is captured using the formulation described below, while the sub-filter contribution is ignored. This is similar to the approach of Wang et al. [21], and has been shown to adequately capture differential diffusion effects, especially when the resolution in the near-flame region is sufficient to have an appreciable contribution from the resolved scales.

The differential diffusion effect is incorporated using the approach of McDermott and Pope [36], which modifies the mixing model. For comparison, the conventional PDF approach without differential diffusion is introduced first followed by the approach for incorporating species-specific molecular transport.

\subsubsection{Conventional IEM model}

The conventional model uses a single diffusivity to account for both large scale and small-scale mixing processes. Based on the original formulation of Villermaux and Falk [65], the LES-specific version has been used in a number of previous studies [43, 63, 64]. The conditional diffusion term is written as

$$
\frac{1}{\bar{\rho}} \overline{\nabla \cdot\left(\rho D_{(\alpha)} \nabla \phi_{\alpha}\right) \mid \boldsymbol{\psi}}=-\frac{1}{\tau_{\phi}}\left(\psi_{\alpha}-\widetilde{\phi}_{\alpha}\right)
$$

where $\tau_{\phi}$ is the scalar mixing time-scale. It is noted that in the conventional IEM model, a single representative molecular diffusivity is used for all the species. Using Eqs. (8) and (9), Eq. (7) is typically solved through a Lagrangian Monte Carlo approach [17]. It is 
assumed that the the initial distribution of notional particle number density is uniform in each cell. These particles subsequently evolve in physical and composition spaces with time. The evolution in physical space is through transport equations that use the filtered flow field from the LES solver, while the evolution in composition space is through mixing and reaction. Similar to prior use of this model [43, 63, 64], a single mixture-averaged diffusivity is used for transporting the PDF. The corresponding stochastic differential equations (SDEs) can then be written as

$$
\begin{aligned}
d \boldsymbol{X}^{*}(t) & =\left[\widetilde{\boldsymbol{u}}+\frac{\nabla \bar{\rho}\left(D+D_{t}\right)}{\bar{\rho}}\right]^{*} d t+\sqrt{2\left(D^{*}+D_{t}^{*}\right)} d \boldsymbol{W} \\
d \boldsymbol{\phi}^{*}(t) & =-\frac{1}{\tau_{\phi}^{*}}\left(\boldsymbol{\phi}^{*}-\widetilde{\boldsymbol{\phi}}^{*}\right) d t+\boldsymbol{S}\left(\boldsymbol{\phi}^{*}\right) d t
\end{aligned}
$$

where $\boldsymbol{X}^{*}(t)$ is the instantaneous particle position. The superscript "** on quantities denotes quantities computed at $\boldsymbol{X}^{*}(t)$ through linear interpolation. $\boldsymbol{W}$ is an isotropic, vector-valued Wiener process that emulates diffusion in physical space and uses a single mixture-averaged value at each particle location. $\widetilde{\phi}$ is the Favre-filtered resolved scalar obtained by weighted sum of particle values in a given filter volume. In the conventional IEM model, $D$ is taken to be the mixture-averaged diffusivity, which will be heavily correlated with the diffusivity of nitrogen for combustion processes with air as oxidizer. With the unity Lewis number assumption, the molecular diffusivity is approximated using an empirical function of temperature obtained from the PDF solver and in this work it is calculated using the subroutine TRANLIB available in CHEMKIN [66].

\subsubsection{IEM model with differential diffusion}

The differential diffusion model is incorporated using the approach of McDermott and Pope [36], and follows the implementation discussed elsewhere [19, 21, 36]. Here, the IEM model is modified such that the stochastic diffusion term in physical space is altered to a diffusion in composition space. The new IEM model is given by

$$
\frac{1}{\bar{\rho}} \overline{\nabla \cdot\left(\rho D_{(\alpha)} \nabla \phi_{\alpha}\right) \mid \boldsymbol{\psi}}=-\frac{1}{\tau_{\phi}}\left(\psi_{\alpha}-\widetilde{\phi}_{\alpha}\right)+\widetilde{\mathcal{D}}_{\alpha},
$$

where

$$
\widetilde{\mathcal{D}}_{\alpha} \equiv \frac{1}{\bar{\rho}} \nabla \cdot\left(\bar{\rho} D_{(\alpha)} \nabla \widetilde{\phi}_{\alpha}\right)
$$

is the mean drift term due to differential diffusion. The molecular diffusivity $D_{\alpha}$ of different chemical species is obtained directly from TRANLIB in CHEMKIN [66]. Using the particle method, Eq. (7) with the model Eqs. (8) and (12) is solved and the corresponding SDEs become

$$
\begin{aligned}
d \boldsymbol{X}^{*}(t) & =\left(\widetilde{\boldsymbol{u}}+\frac{\nabla \bar{\rho} D_{t}}{\bar{\rho}}\right)^{*} d t+\sqrt{2 D_{t}^{*}} d \boldsymbol{W} \\
d \boldsymbol{\phi}^{*}(t) & =-\frac{1}{\tau_{\phi}^{*}}\left(\boldsymbol{\phi}^{*}-\widetilde{\boldsymbol{\phi}}^{*}\right) d t+\widetilde{\mathcal{D}}_{\alpha}^{*} d t+\boldsymbol{S}\left(\boldsymbol{\phi}^{*}\right) d t .
\end{aligned}
$$


As noted by McDermott and Pope [36], this implementation does not give rise to the spurious production of scalar variance. Nevertheless, some numerical issues arise due to the inclusion of differential diffusion in Eq. (15), and the algorithm provided in that work is used here.

\subsubsection{Local dynamic model for mixing time-scale}

Typically, a constant ratio of scalar mixing time-scale $\tau_{\phi}$ to turbulent time-scale $\tau$ is assumed. The scalar mixing time-scale in Eqs. (9) and (12) is evaluated such that

$$
\tau_{\phi}=\tau / C_{\phi}=\frac{\Delta^{2}}{C_{\phi}\left(D+D_{t}\right)}
$$

where $\Delta$ is the local filter width and $C_{\phi}$ is the constant ratio for all the composition. However, the assumption that $C_{\phi}=2.0$ is valid only when both the scalar and velocity spectra are at equilibrium. In jet flows, such equilibrium is reached only at far downstream locations. Consequently, this parameter can have substantial variations and can strongly influence the combustion process. In previous LES/PDF calculations, a wide range of values has been used $[1,17,19,67]$.

An alternative approach is to treat this parameter as a spatially and temporally varying quantity. In this case, a local dynamic model for the time-scale can be formulated [62], which removes the need to specify the constant time-scale ratio. The local dynamic model is obtained from the dynamic models for the sub-filter variance and the scalar dissipation rate of a conserved scalar $Z$. The commonly used models for the conserved scalar variance and dissipation rate have the following form [61]:

$$
\widetilde{Z^{\prime \prime 2}}=C_{Z} \Delta^{2} \nabla \widetilde{Z} \cdot \nabla \widetilde{Z}
$$

and

$$
\chi=\left(D+D_{t}\right) \nabla \widetilde{Z} \cdot \nabla \widetilde{Z}
$$

where $D_{t}$ and $C_{Z}$ are both obtained dynamically as follows [61]:

$$
D_{t}=\frac{\left\langle\mathcal{L}_{i} \mathcal{M}_{i}\right\rangle}{\left\langle\mathcal{M}_{j} \mathcal{M}_{j}\right\rangle} \Delta^{2}|\widetilde{S}| \quad \text { and } \quad C_{Z}=\frac{\langle\mathcal{L} \mathcal{M}\rangle}{\left\langle\mathcal{M}^{2}\right\rangle}
$$

in which $\mathcal{L}$ is called the Leonard term, $\mathcal{M}$ is the model term, and $|\widetilde{S}|=\sqrt{\widetilde{S}_{i j} \widetilde{S}_{i j}}$

Using these relations, the scalar mixing time-scale can be locally and dynamically computed as

$$
\tau_{\phi}=\widetilde{Z^{\prime \prime 2}} / \chi=\frac{\Delta^{2}}{C_{Z}^{-1}\left(D+D_{t}\right)} .
$$

It is seen that the difference between Eq. (16) and Eq. (20) is that $C_{\phi}$ is a constant while $C_{Z}$ is dynamically evaluated in the simulations. It is noted that using Eq. (20), the individual (and possibly) more accurate mixing time-scales for all compositions can be computed when corresponding the scalar dissipation rates are provided. The advantage of 
using a conserved scalar to estimate mixing time-scale is that its scalar dissipation rate can be modeled based on the assumption that production equals dissipation in the conserved scalar variance equation. For non-conserved scalars, Eq. (18) should be modified to include the co-variance of scalar and scalar production rate appearing in the reactive scalar variance equation. For simplicity and ensuring numerical stability, the mixing time-scales for all composition are approximated by that of the conserved scalar in the present work.

\subsection{Two-way coupling algorithm}

The coupling between the low-Mach number LES solver and Monte Carlo PDF solver should be treated carefully. The unsteady LES solver requires that any solver that is coupled be time-accurate as well. The LES solver provides the velocity, turbulent diffusivity, and scalar mixing time-scale to the PDF solver which returns the density field and transport properties. However, the gas-phase density, directly obtained from the PDF solver, contains statistical noise due to the finite number of particles per filter volume, which can give rise to numerical instabilities. To address the issue, an equivalent-enthalpy $(\hbar)$ transport equation $[43,68]$ is solved as follows:

$$
\frac{\partial \bar{\rho} \widetilde{\hbar}}{\partial t}+\frac{\partial \bar{\rho} \widetilde{u}_{j} \widetilde{\hbar}}{\partial x_{j}}=\frac{\partial}{\partial x_{j}}\left(\bar{\rho}\left(D+D_{t}\right) \frac{\partial \widetilde{\hbar}}{\partial x_{j}}\right)+\bar{\rho} \widetilde{S_{\hbar}}
$$

with

$$
\widetilde{S_{\hbar}}=\widetilde{\frac{\partial \hbar}{\partial \phi_{\alpha}} S_{\alpha}}-\frac{1}{2 \tau_{\phi}} \widetilde{\frac{\partial \hbar}{\partial \phi_{\alpha}} \phi_{\alpha}^{\prime \prime}}
$$

which represents the source term due to reaction and mixing $[43,68]$. This source term can be computed directly from the PDF solver.

Once the equivalent enthalpy is known, the new density is computed using the ideal gas law:

$$
\bar{\rho}=\frac{\gamma}{\gamma-1} \frac{P_{0}}{\widetilde{\hbar}}
$$

where $P_{0}$ is background pressure, and $\gamma$ is the local ratio of specific heat capacities. The present two-way coupling algorithm is verified by examining the consistency between the LES and PDF mean density (see Appendix A).

In addition, to obtain the scalar mixing time-scale $\tau_{\phi}$, a transport equation for mixture fraction is solved along with the other LES transport equations:

$$
\frac{\partial \bar{\rho} \widetilde{Z}}{\partial t}+\frac{\partial \bar{\rho} \widetilde{u}_{j} \widetilde{Z}}{\partial x_{j}}=\frac{\partial}{\partial x_{j}}\left(\bar{\rho}\left(D+D_{t}\right) \frac{\partial \widetilde{Z}}{\partial x_{j}}\right)
$$

It is noted that the source term arising from differential diffusion in the transport equation of mixture fraction is neglected. The third-order BQUICK scheme [69] is used to discretize the nonlinear terms in the scalar transport equations (21) and (24). 


\section{Flow configuration}

The experiment used for validating the LES/PDF approach corresponds to the vitiated coflow burner (Fig. 1) that was experimentally studied by Cabra et al. [10]. Using this burner, $\mathrm{Wu}$ et al. [45] investigated the effect of coflow temperature, jet and coflow velocities on the lift-off height. This flame has been modeled using the RANS framework and transported-PDF methods [10, 22, 23, 26, 46-51]. Conditional-moment closure (CMC) based results were reported in [52]. LES calculations were reported in [24] and [53], in which the turbulence-chemistry interactions were modeled using Eulerian stochastic field methods and perfectly stirred reactors, respectively. Recently, LES-CMC calculations were performed $[54,55]$, while RANS/UFPV calculations were reported in [15]. The focus here is on the impact of differential diffusion, which is analyzed using the LES/PDF approach discussed in the previous sections.

A schematic of the burner is provided in Fig. 1. The burner consists of a central fuel pipe with a diameter of $D_{\text {jet }}=4.57 \mathrm{~mm}$, in which a 1:3 ratio (by volume) of $\mathrm{H}_{2} / \mathrm{N}_{2}$ mixture at a temperature of $305 \mathrm{~K}$ and exit bulk velocity of $U_{\text {jet }}=107 \mathrm{~m} / \mathrm{s}$ is supplied. The vitiated coflow is composed of hot combustion products of lean premixed $\mathrm{H}_{2}$ /air. Experimental results show that the products, $\mathrm{O}_{2}, \mathrm{H}_{2} \mathrm{O}$, and $\mathrm{N}_{2}$ are uniform across the coflow stream with temperature of $1045 \mathrm{~K}$. The coflow is stabilized by a disk with a diameter of $210 \mathrm{~mm}$. The disk has a $87 \%$ blockage and is made of 2200 holes, each with a diameter of $1.58 \mathrm{~mm}$ to burn the premixed $\mathrm{H}_{2}$ /air mixture. An exit collar surrounds the perforated disk to prevent entrainment of ambient air into the flame. The Reynolds number based on the jet diameter, exit velocity, and kinematic viscosity of the fuel mixture is 23600, and the stoichiometric mixture fraction based on Bilger's formulation is $Z_{s t}=0.474$. The observed lift-off height $H$ was about $10 D_{j e t}$. The boundary conditions for species and temperature are summarized in Table 1.

\section{Simulation details}

This LES/PDF is performed in a cylindrical coordinate system $(x, r, \theta)$. Starting at the fuel jet exit, a computational domain of $L_{x} \times L_{r} \times L_{\theta}=30 D_{\text {jet }} \times 15 D_{\text {jet }} \times 2 \pi$ is used in the axial, radial and azimuthal directions, respectively, with a non-uniform stretched grid of size $N_{x} \times N_{r} \times N_{\theta}=192 \times 108 \times 32$. A stretched grid is used to resolve the central jet and the shear layers. The turbulent jet boundary condition is obtained from a separate pipe simulation at the experimental mass flow rate. The velocity boundary condition for the coflow stream is evaluated according to the formulation in [15]. Previous numerical studies [23] have shown that the predictions are insensitive to coflow velocity, jet temperature and jet velocity. In addition to this baseline grid, two other calculations with a coarser and a refined grid were performed to ensure that grid resolution did not affect nature of the results (See Appendix B). It should be noted that grid-filtered LES will always be sensitive to the choice of grid spacing, but its sensitivity can be understood by conducting multiple simulations.

The PDF transport equation is solved using the Lagrangian Monte Carlo approach. Each computational cell is allocated 20 notional particles, and particle count is constrained 
such that it ranges from 12-40 per cell. This range is enforced using merging and splitting techniques [43]. The gas phase chemistry was described using the mechanism of Li et al. [70]. It involves 9 chemical species and 21 elementary reactions. The ISAT algorithm is applied to accelerate the computations [57]. The effect of the ISAT and ODE error tolerances on the prediction of the particular lifted flame has been studied systematically by Masri et al. [22]. Since essentially the same ISAT and chemical mechanism are implemented in the present work, the same parameters, i.e., ISAT and ODE error tolerances of $6.25 \times 10^{-6}$ and $1 \times 10^{-8}$, respectively, are employed here. Cao et al. [23] also used an identical set of parameters. For this particular Cabra flame, previous studies [22, 23] have shown that the tabulation error results in less than $2 \%$ error in the conditional mean temperature at the stoichiometric mixture fraction, a quantity which is found to be very sensitive to such errors. To achieve parallel scale-up, the particles are grouped based on mixture fraction and distributed amongst processors. Each processor thus builds a tree that is nearly local in composition space, further reducing the ISAT tabulations. This grouping and re-distribution is updated at each time-step. A scale-up of 5-10 times was achieved compared to building ISAT tables independently on each processor.

In the enhanced LES/PDF/ISAT approach, there is no need to specify the value of model constant in the IEM model due to the implementation of a dynamic model for the mixing time-scale. The uncertainties of calculations are mainly from the initial number of notional particles $\left(\mathrm{N}_{\mathrm{pc}}\right)$ in each computational cell and the choice of filter size. In the present study, the grid convergence study is conducted (See Appendix B) in order to ensure the present filter size are accurate enough (numerically) to analyze the effect of differential diffusion. Furthermore, [71] has demonstrated that the bias error due to the number of particles per cell scales as $\frac{1}{N_{\mathrm{pc}}}$. Hence, typically $\mathrm{N}_{\mathrm{pc}}=20$ notional particles are used in each cell in the context of LES based on the consideration of cost and accuracy.

This LES/PDF/ISAT simulation was carried out on a distributed memory parallel machine with 192 processors. For the LES/PDF/ISAT simulation, the jet reached the statistically stationary state at $t / T_{\text {jet }} \approx 20$, where $T_{\text {jet }}=L_{x} / U_{\text {jet }} \approx 1.3 \mathrm{~ms}$ is the flow-through time. For each case, statistics were collected over 30 flow-through times.

For the discussion of results below, four different cases were considered (see Table 2). In Case 1, the differential diffusion effect is not included in the IEM model and the scalar mixing time-scale is evaluated via Eq. (16). Case 2 also neglects differential diffusion but uses the dynamic model for the mixing time-scale. In Case 3, both molecular transport of all species and dynamic mixing time-scale model are considered. Case 4 is similar to Case 3 but neglects the molecular transport of $\mathrm{H}$ and $\mathrm{H}_{2}$ by setting $D_{\mathrm{H}}=D_{\mathrm{H}_{2}}=0$. The flame sensitivity to scalar mixing time-scale can be analyzed by comparison between Case 1 and Case 2. The flame sensitivity to differential diffusion can be analyzed by comparison between Case 2 and Case 3. In addition to these cases, a series of simulations that vary the coflow temperature was also conducted in order to assess the sensitivity of the lift-off height predictions to coflow boundary conditions. For this analysis, two sets of simulations with conditions corresponding to Case 2 and 3 were used. 


\section{Results and discussion}

\subsection{Instantaneous results}

The instantaneous data below is shown to identify the progress towards a statistically stationary state starting from initial conditions. Initially, chemical reactions are turned off and the simulations run until steady state is reached. After this, chemical reactions and related heat release source terms are included. Figure 2 shows that the ignition zone is first located downstream, where sufficiently low strain combined with adequate time for auto-ignition leads to the formation of the turbulent flame. These early ignition kernels then transition upstream due to diffusion of temperature that can overcome the shorter residence time as well as larger strain caused by larger turbulence intensity. Eventually, the flame is stabilized at a lift-off height. Similar to $[10,23]$, the stationary lift-off height is defined as the axial location where time-averaged $\mathrm{OH}$ mass fraction reaches $2 \times 10^{-4}$ at any radius. There are no obvious autoignition events below the lift-off height once a statistically stationary condition is reached. Figure 2 also shows that the $\mathrm{OH}$ mass fraction is present largely in the mixing layer between central jet and coflow, which indicates the autoignition is the main stabilization mechanism of the lifted flame as suggested in [10, 23, 24, 55]. It should be noted that the instantaneous lift-off height based on the instantaneous $\mathrm{OH}$ mass fraction does fluctuate in time in a range of 3-4 jet diameters around the time-averaged lift-off height with a frequency of approximately $200 \mathrm{~Hz}$ due to random and chaotic time dependent turbulence. This is consistent with experimental observations of lift-off height fluctuations [26], where fluctuations of 4-5 jet diameters were reported.

Figure 3 shows the time-averaged species-specific molecular diffusivities and the turbulent diffusivity for two different axial locations. At both locations, it is seeen that the turbulent diffusivity is much smaller than the molecular diffusivity, especially that of the fast-diffusing $\mathrm{H}_{2}$ and $\mathrm{H}$ species. Near the edge of the main jet, turbulent diffusivity is comparable to the diffusivities of the heavier molecules. These results clearly indicate that molecular diffusivity is important near the flame stabilization zone, and cannot be neglected in the simulation of such flames.

The effect of differential diffusion on the instantaneous fields of temperature and $\mathrm{OH}$ are shown in Fig. 4. It is seen that autoignition in the radial direction mainly happens within the region enclosed by the stoichiometric mixture fraction and the most reactive mixture fraction. Here, the most reactive mixture fraction is defined as the mixture fraction that is most likely to ignite [72]. It is noted that in Ref. [46], the most reactive mixture fraction is defined by considering different coflow temperatures within the context of Cabra's configuration where the micro-mixing is considered. The shortest ignition delay time occurs around $Z=0.045 \sim 0.052$. In the present work, however, the most reactive mixture fraction is determined with a homogeneous reactor in CHEMKIN III [73] where the micro-mixing is switched off, giving thus a parallel solution to a series of homogeneous reactors of variable mixture fraction. We found that the shortest ignition delay time is reached at $Z \approx 0.054$ when initial temperature is $1070 \mathrm{~K}$ for updated $\mathrm{H}_{2}$ mechanism [70]. The small difference between the two methods is mainly caused by the different computational configurations and different reaction mechanisms. Figure 4 shows that including the differential diffusion 
effect decreases the lift-off height and the thickness of autoignition zone. Figure 4 indicates a lift-off height of $H / D_{\text {jet }} \approx 10$ for flames with differential diffusion and $H / D_{\text {jet }} \approx 13$ for flames without differential diffusion. This is partially attributed to the fact that the increase in mixing due to differential diffusion serves to enhance combustion by increasing the probability of ignition kernels surviving turbulent mixing in this system. Cabra et al. [10] reported the experimentally observed lift-off height was $H / D_{\text {jet }} \approx 10$. In previous studies, the lower [23, 24, 52] or higher [15] experimental coflow temperature of $1045 \mathrm{~K}$ was adopted to obtain the the lift-off height of $10 D_{\text {jet }}$. Therefore, the simulations considering differential diffusion appear to predict the experimental lift-off with the experimental coflow temperature.

\subsection{Time-averaged statistics}

For Case 1, the mixing model coefficient $C_{\phi}$ needs to be specified. Here, several simulations with values ranging from 2 to 20 were performed (see Appendix C), with the optimal value found to be 10 . This optimal value implies that the profiles are as close to experiments as possible by changing the parameter. In Case 2, the model coefficient is determined dynamically and need not be specified a priori. The radial profiles of time-averaged $C_{Z}^{-1}$ (which is dynamically-determined $C_{\phi}$ ) at different axial locations for Case 2 are investigated (see Appendix C). Figures 5 and 6 show variations in flame statistics, denoted by $\langle\cdot\rangle$, to the scalar mixing time-scale at three different axial locations. Overall, good agreement with experiments is observed. The slight difference stems from the fact that the early stages of ignition occurs at $x \approx 10 D_{\text {jet }}$ in the measurements while the simulations predict ignition farther downstream. As a result, the mean profiles of mixture fraction $Z$ and mass fraction of $\mathrm{H}_{2}$ are overpredicted and temperature $T$ and mass fraction of $\mathrm{OH}$ are underpredicted. The comparison between Case 1 and Case 2 shows that the local dynamic model for mixing time-scale can provide results consistent with the optimal value of $C_{\phi}$. Since the model is dynamic in nature, it can also adjust to any changes in flame structure based on the solution at the current time-step. Hence, for Case 3 and 4, the dynamic model is used to describe the mixing time.

The effect of differential diffusion is analyzed in Figs. 7-20. The prediction of the farthest downstream location measured in the experiment $x / D_{\text {jet }}=26$ is also presented (see Appendix D). The results of Case 4 are included for identifying the critical species that influence flame stabilization. Figures 7-14 compare the radial profiles of mean and RMS fluctuations of mixture fraction, temperature, and mass fractions of fuel and oxygen for

Cases 2-4 with experimental data. It is seen that the mean and RMS profiles are in good overall agreement with experimental data for Case 3. The mixture fraction profiles are insensitive to the differential diffusion models utilized, since it is assumed to be a conserved scalar in the present work and the source term arising from differential diffusion in the transport equation of mixture fraction is neglected for simplicity. The radial profiles of mean and RMS of temperature show that including differential diffusion effects considerably alters the results. In particular, the prediction of ignition as well as the radial temperature profiles are significantly better when both differential diffusion and dynamic mixing scales are used. Interestingly, reverting to a constant mixing coefficient or setting diffusivity of hydrogen 
species to zero result in similar profiles for temperature. This indicates that the main role of differential diffusion is in enhancing flame ignition, which causes the stabilization point to move further upstream towards the inlet. The increase/decrease in temperature/mass fraction of $\mathrm{H}_{2}$ is more distinct at $x / D=11,14$ due to the increased role of molecular diffusivity at these downstream locations.

Comparisons between Case 3 and 4 reveal that the diffusion of both $\mathrm{H}$ and $\mathrm{H}_{2}$ play an important role in flame stabilization. Since the stabilization mechanism of the lifted flame is due to autoignition chemistry, the mass transport of $\mathrm{H}_{2}$ (involved in the chain initiation reaction $\mathrm{H}_{2}+\mathrm{O}_{2}=\mathrm{HO}_{2}+\mathrm{H}$ ) and $\mathrm{H}$ (involved in the chain branching reaction $\mathrm{H}+\mathrm{O}_{2}=\mathrm{OH}+\mathrm{O}$ ) are very important. The molecular transport of $\mathrm{H}_{2}$ and $\mathrm{H}$ can improve the probability of autoignition events and hence increase the $\mathrm{H}_{2}$ consumption and temperature, which can be seen from the comparisons between Cases 3 and 4 in Figs 7-14. It is noted that the differential diffusion effect is negligible at $r / D_{\text {jet }}>4$.

Radial profiles of mass fractions of intermediate species $\mathrm{OH}$ and product $\mathrm{H}_{2} \mathrm{O}$ are illustrated in Figs. 15 and 18. The $\mathrm{OH}$ mass fraction plot shows that the predicted $Y_{\mathrm{OH}}$ is considerably lower than the experimental data for Cases 2 and 4 , especially at $x / D_{\text {jet }}=10$. This is attributed to the lift-off heights of Cases 2 and 4 are overpredicted and the temperature is underpredicted, as shown in Figs. 4 and 9. Similar to Figs 7-14, it is seen that the mean and RMS profiles, computed with differential diffusion, are in good agreement with experimental data. This is due to the fact $\mathrm{H}_{2}$ and $\mathrm{H}$ transport in the mixture have a great effect on the $\mathrm{OH}$-formation reaction $\left(\mathrm{H}_{2}+\mathrm{O}_{2}=\mathrm{HO}_{2}+\mathrm{H}\right.$ and $\left.\mathrm{H}+\mathrm{O}_{2}=\mathrm{OH}+\mathrm{O}\right)$, especially at the flame base. Similar to $Y_{\mathrm{OH}}$ prediction, Fig. 17 shows Cases 2 and 4 underpredict the mean $\mathrm{H}_{2} \mathrm{O}$ mass fraction and calculations with differential diffusion (Case 3) leads to improved results.

Figures 19 and 20 show the radial profiles of mean and RMS of $\mathrm{N}_{2}$ mass fraction at different axial locations, which confirms the observations about mixture fraction (Fig. 7). It is seen that no obvious difference is found for mean mass fraction of $\mathrm{Y}_{\mathrm{N}_{2}}$ between the three cases. Since $\mathrm{N}_{2}$ is inert in these calculations, its evolution mainly depends on the flow field. Nevertheless, it is noted that $\mathrm{Y}_{\mathrm{N}_{2}}$ is not a conserved scalar since it is affected by differential diffusion between species. Therefore, unlike mixture fraction that is assumed to be a conserved scalar in the present work, it is found that considering differential diffusion can improve the variance prediction of $\mathrm{Y}_{\mathrm{N}_{2}}$, which further suggests the difference between the natures of $\mathrm{N}_{2}$ and mixture fraction in the present work.

From the above discussion, it can be concluded that species mass fractions and temperature are considerably affected by including differential diffusion physics. Case 3 yields significantly improved predictions and provides the best agreement with experimental data. Further, the effect of differential diffusion is nearly absent beyond a radius of $4 D_{\text {jet }}$.

\subsection{Conditional statistics}

To further analyze the sensitivity of the lifted flames to differential diffusion, Figs. 21 and 22 show scatter data and conditional means of temperature and mass fraction of $\mathrm{OH}$ at two axial locations $\left(x / D_{\text {jet }}=10,11\right)$ around the flame base for Cases 2 and 3 . These two locations correspond to regions where both mixing and chemical reactions leading to ignition 
are important. When the central fuel jet entrains hot oxidizer coflow, mixing is dominant at $x / D_{\text {jet }}=10$ as seen by the scatter points distributed close to the mixing line. However, there are some points away from the mixing line indicating limited ignition behavior. As the core of the fuel jet breaks down, the mixture fraction of fuel-rich side at $x / D_{\text {jet }}=10$ decreases from $Z=1$ to values between $Z \sim 0.9$ and $Z \sim 0.8$, such that autoignition events happen. At $x / D_{\text {jet }}=11$, one diameter above the lift-off height, it is interesting to find that the data is scattered throughout the zone enclosed by the pure mixing line and fast chemistry limit. In a time-averaged sense, this would imply the existence of a thickened flame brush near the flame base. In this region, it is likely then that subgrid fluctuations will play a role in establishing the flame. It is apparent from Fig. 21 that considering differential diffusion can increase the conditional temperature which is consistent with the good prediction of unconditional temperature shown in Fig. 9. Figure 22 shows that the conditional mean of $Y_{\mathrm{OH}}$ is considerably increased when differential diffusion effects are included, resulting in the lift-off height of $H / D_{\text {jet }} \approx 10$. It is seen that the prediction of $Y_{\mathrm{OH}}$ is very sensitive to differential diffusion effect in the present LES/PDF study, which is consistent with the prediction mean $Y_{\mathrm{OH}}$ in Fig. 15. The above discussions indicates the enhanced PDF model that includes differential diffusion and local dynamic model for mixing time-scale is capable of capturing the small-scale ignition behavior.

\subsection{Sensitivity of lift-off height to coflow temperature}

Due to the importance of coflow temperature on the ignition process, the lift-off height is highly sensitive to the coflow boundary conditions. Previous experiments and simulations have demonstrated that even $60-100 \mathrm{~K}$ difference in coflow temperature can triple the liftoff height $[23,45]$. To verify that the LES/PDF calculations are able to reproduce this sensitivity, a number of cases with different coflow temperatures were computed, and the results are shown in Fig. 23. The simulations used models corresponding to Case 2 and Case 3 with a jet velocity of $107 \mathrm{~m} / \mathrm{s}$. The three sets of experimental data, obtained by Cabra et al. [10], Wu et al. [45], and Gordon et al. [26], were measured at different times with different thermocouples, and the differences between the experimental data are within the expected error due to the uncertainty in the temperature measurement which, at these temperatures, is of the order of $30 \mathrm{~K}$ [10]. Figure 23 shows that the flame is essentially attached to the burner when the coflow temperature is larger than $1080 \mathrm{~K}$. Lifted flames can be observed as the temperature decreases from $1080 \mathrm{~K}$. The lift-off height strongly depends on the coflow temperature such that a slight change in coflow temperature can result in a considerable increase in the lift-off height. It is found that the present predictions are generally consistent with the most recent results obtained by an unsteady flamelet/progress variable (UFPV) approach within the RANS framework [15]. It is noted that the coflow temperature in Ref. [15] is increased to $1062 \mathrm{~K}$ to match the experimental data measured by Cabra et al. [10], but in this work the lift-off height $H / D_{\text {jet }} \approx 10$ is accurately predicted by including the differential diffusion effect (Case 3) at the coflow temperature of $1405 \mathrm{~K}$ without any adjustment. In previous simulations [23, 24, 26, 49, 52], in order to obtain the same lift-off height the coflow temperature had to be reduced to about $1030 \mathrm{~K}$. Comparisons between Cases 2 and 3 shows that accounting for the differential diffusion decreases the lift-off height, 
which results in good agreement with measurements by Gordon et al. [26]. This indicates that differential diffusion has an effect on the lift-off height.

\section{Conclusions}

LES/PDF computations of autoignition in lifted turbulent flames were performed. The PDF method was extended to account for differential diffusion effect, while a dynamic scalar mixing time-scale was used to describe small scale mixing. The ISAT algorithm was applied to accelerate the chemistry calculations. The autoignition-stabilized $\mathrm{H}_{2} / \mathrm{N}_{2}$ lifted turbulent flames in the vitiated coflow burner were studied using the LES/PDF/ISAT approach. The flame sensitivity to differential diffusion and scalar mixing time-scale were thoroughly assessed. The following conclusions can be drawn based on these studies:

- Compared to the standard IEM model in the PDF method, the IEM model with differential diffusion and dynamic model for scalar mixing time-scale provides better predictions of the major species mass fractions, temperatures and lift-off heights of the lifted turbulent flames.

- The predictions of temperatures, species mass fractions, and lifted-off height are very sensitive to differential diffusion. It is found that $\mathrm{H}_{2}$ and $\mathrm{H}$ are two critical species that play a dominant role in this flame due to their high diffusivity and importance in the chain initiation and branching reactions.

- Although the dynamic model for mixing time-scale removes the need to specify the value of model constant in the IEM model, it only slightly improves the predictions than using the optimal value of model constant. Nevertheless, the dynamic approach is robust and cost-effective, since the optimal value can be determined only based on multiple simulations and comparisons with experimental data.

- The presence of differential diffusion enhances autoignition events near the base of the flame, which is the key mechanism by which flame stabilization is achieved. However, the presence of differential diffusion makes the flame narrower in physical space, which is consistent with the experimental data as well. This is mainly due to localized heat release that increase fluid viscosity/diffusivity, which reduces turbulent fluctuations.

Given these results, this enhanced LES/PDF/ISAT approach is seen as a promising tool for modeling similar flame configurations where small-scale molecular transport, turbulent mixing, and kinetics are all important. For instance, flames operating in the MILD combustion regime, flames with local extinction and operating close to blowout will benefit from this modeling approach. To further demonstrate the robustness of the enhanced LES/PDF/ISAT approach, it is necessary to perform such comparisons with experiments for a range of lifted flames, and this will be the focus of future work. 


\section{Acknowledgements}

WH would like to thank China Scholarship Council (CSC) scholarship (No. 201506010207) for supporting this study at the University of Michigan. VR gratefully acknowledges financial support from the US National Science Foundation through grant CBET-1502477. ZC would like to thank the support from National Natural Science Foundation of China (Nos. 51322602 and 91541204). Helpful discussions with Mr. Malik Hassanaly, Dr. Heeseok Koo and Dr. Colin Heye are appreciated. This work also greatly benefited from discussions with Dr. Bertrand Naud who shared with the authors the experimental data of Ref. [10]. The authors also gratefully acknowledge the generous allocation of computing time by the Texas Advanced Computing Center.

\section{References}

[1] N. Peters, Turbulent Combustion, Cambridge University Press, Cambridge, 2000.

[2] C.K. Law, Combustion Physics, Cambridge University Press, Cambridge, 2006.

[3] T. Poinsot, D. Veynante, Theoretical and Numerical Combustion, Edwards, Philadelphia, 2005.

[4] A. Liñán, M. Vera, S.L. Antonio, Ignition, liftoff, and extinction of gaseous diffusion flames, Annu. Rev. Fluid Mech. 47 (2015) 293-314.

[5] Y. Mizobuchi, S. Tachibana, J. Shinio, S. Ogawa, T. Takeno, A numerical analysis of the structure of a turbulent hydrogen jet lifted flame, Proc. Combust. Inst. 29 (2002) 2009-2015.

[6] Y. Mizobuchi, J. Shinjo, S. Ogawa, T. Takeno, A numerical study on the formation of diffusion flame islands in a turbulent hydrogen jet lifted flame, Proc. Combust. Inst. 30 (2005) 611-619.

[7] C.S. Yoo, E.S. Richardson, R. Sankaran, J.H. Chen, A DNS study on the stabilization mechanism of a turbulent lifted ethylene jet flame in highly-heated coflow, Proc. Combust. Inst. 33 (2011) 1619-1627.

[8] C.S. Yoo, R. Sankaran, J.H. Chen, Three-dimensional direct numerical simulation of a turbulent lifted hydrogen jet flame in heated coflow: flame stabilization and structure, J. Fluid Mech. 640 (2009) 453-481.

[9] S. Deng, P. Zhao, M.E. Mueller, C.K. Law, Autoignition-affected stabilization of laminar nonpremixed DME/air coflow flames, Combust. Flame 162 (2015) 3437-3445.

[10] R. Cabra, T. Myhrvold, J.Y. Chen, R.W. Dibble, A.N. Karpetis, R.S. Barlow, Simultaneous laser raman-rayleigh-lif measurements and numerical modeling results of a lifted turbulent $\mathrm{H}_{2} / \mathrm{N}_{2}$ jet flame in a vitiated coflow, Proc. Combust. Inst. 29 (2002) 1881-1888.

[11] R. Cabra, J.Y. Chen, R.W. Dibble, A.N. Karpetis, R.S. Barlow, Lifted methane-air jet flames in a vitiated coflow, Combust. Flame 143 (2005) 491-506.

[12] C.N. Markides, E. Mastorakos, An experimental study of hydrogen autoignition in a turbulent co-flow of heated air, Proc. Combust. Inst. 30 (2005) 883-891.

[13] H. Pitsch, Large-eddy simulation of turbulent combustion, Annu. Rev. Fluid Mech. 38 (2006) 453-482.

[14] M. Ihme, Y.C. See, Prediction of autoignition in a lifted methane/air flame using an unsteady flamelet/progress variable model, Combust. Flame 157 (2010) 1850-1862.

[15] B. Naud, R. Novella, J.M. Pastor, J.F. Winklinger, RANS modelling of a lifted $\mathrm{H}_{2} / \mathrm{N}_{2}$ flame using an unsteady flamelet progress variable approach with presumed PDF, Combust. Flame 162 (2015) 893-906.

[16] S.B. Pope, PDF methods for turbulent reactive flows, Prog. Energy Combust. Sci. 11 (1985) 119-192.

[17] D.C. Haworth, Progress in probability density function methods for turbulent reacting flows, Prog. Energy Combust. Sci. 36 (2010) 168-259.

[18] S.B. Pope, Small scales, many species and the manifold challenges of turbulent combustion, Proc. Combust. Inst. 34 (2013) 1-31.

[19] Y. Yang, H. Wang, S.B. Pope, J.H. Chen, Large-eddy simulation/probability density function modeling of a non-premixed $\mathrm{CO} / \mathrm{H}_{2}$ temporally evolving jet flame, Proc. Combust. Inst. 34 (2013) 1241-1249. 
[20] H. Wang, M. Juddoo, S.H. Starner, A.R. Masri, S.B. Pope, A novel transient turbulent jet flame for studying turbulent combustion, Proc. Combust. Inst. 34 (2013) 1251-1259.

[21] H. Wang, K. Kim, Effect of molecular transport on PDF modeling of turbulent non-premixed flames, Proc. Combust. Inst. 35 (2015) 1137-1145.

[22] A.R. Masri, R. Cao, S.B. Pope, G.M. Goldin, PDF calculations of turbulent lifted flames of $\mathrm{H}_{2} / \mathrm{N}_{2}$ fuel issuing into a vitiated co-flow, Combust. Theor. Model. 8 (2004) 1-22.

[23] R. Cao, S.B. Pope, A.R. Masri, Turbulent lifted flames in a vitiated coflow investigated using joint PDF calculations, Combust. Flame 142 (2005) 438-453.

[24] W.P. Jones, S. Navarro-Martinez, Large eddy simulation of autoignition with a subgrid probability density function method, Combust. Flame 150 (2007) 170-187.

[25] R.L. Gordon, A.R. Masri, S.B. Pope, G.M. Goldin, Transport budgets in turbulent lifted flames of methane autoigniting in a vitiated co-flow, Combust. Flame 151 (2007) 495-511.

[26] R.L. Gordon, A.R. Masri, S.B. Pope, G.M. Goldin, A numerical study of auto-ignition in turbulent lifted flames issuing into a vitiated co-flow, Combust. Theor. Model. 11 (2007) 351-376.

[27] Y. Yang, S.B. Pope, J.H. Chen, An LES/PDF study of a turbulent lifted ethylene jet flame in a heated coflow, 8th U.S. National Combustion Meeting 20132 (2013) 913-920.

[28] C. Heye, V. Raman, A.R. Masri, Influence of spray/combustion interactions on auto-ignition of methanol spray flames, Proc. Combust. Inst. 35 (2015) 1639-1648.

[29] E.S. Richardson, J.H. Chen, Application of PDF mixing models to premixed flames with differential diffusion, Combust. Flame 159 (2012) 2398-2414.

[30] E. Hawkes, A. Krisman, Modelling of DNS cases, 12th International Workshop on Measurement and Computation of Turbulent Flames (2014) 346-349.

[31] J.D. Regele, E. Knudsen, H. Pitsch, G. Blanquart, A two-equation model for non-unity lewis number differential diffusion in lean premixed laminar flames, Combust. Flame 160 (2013) 240-250.

[32] S.B. Pope, Turbulent Flows, Cambridge University Press, Cambridge, 2000.

[33] R.O. Fox, Computational Models for Turbulent Reacting Flows, Cambridge University Press, Cambridge, 2003.

[34] K.A. Kemenov, H. Wang, S.B. Pope, Modelling effects of subgrid-scale mixture fraction variance in LES of a piloted diffusion flame, Combust. Theor. Model. 16 (2012) 611-638.

[35] H. Pitsch, Unsteady flamelet modeling of differential diffusion in turbulent jet diffusion flames, Combust. Flame 123 (2000) 358-374.

[36] R. McDermott, S.B. Pope, A particle formulation for treating differential diffusion in filtered density function methods, J. Comput. Phys. 226 (2007) 947-993.

[37] A.J. Aspden, M.S. Day, J.B. Bell, Lewis number effects in distributed flames, Proc. Combust. Inst. 33 (2011) 1473-1480.

[38] B. Savard, G. Blanquart, Broken reaction zone and differential diffusion effects in high Karlovitz n$\mathrm{C}_{7} \mathrm{H}_{16}$ premixed turbulent flames, Combust. Flame 162 (2015) 2020-2033.

[39] S. Lapointe, B. Savard, G. Blanquart, Differential diffusion effects, distributed burning, and local extinctions in high Karlovitz premixed flames, Combust. Flame 162 (2015) 3341-3355.

[40] D. Frederick, J.Y. Chen, Effects of differential diffusion on predicted autoignition delay times inspired by $\mathrm{H}_{2} / \mathrm{N}_{2}$ jet flames in a vitiated coflow using the linear eddy model, Flow Turbul. Combust. 93 (2014) 283-304.

[41] C. Celis, L.F. Figueira da Silva, Lagrangian mixing models for turbulent combustion: review and prospects, Flow Turbul. Combust. 94 (2015) 643-689.

[42] S.B. Pope, Ten questions concerning the large-eddy simulation of turbulent flows, New J. Phys. 6 (2004) p. 35.

[43] V. Raman, H. Pitsch, R.O. Fox, Hybrid large-eddy simulation/lagrangian filtered-density-function approach for simulating turbulent combustion, Combust. Flame 143 (2005) 56-78.

[44] H. Wang, Consistent flamelet modeling of differential molecular diffusion for turbulent non-premixed flames, Phys. Fluids 28 (2016) 035102.

[45] Z. Wu, A.R. Masri, R.W. Bilger, An experimental investigation of the turbulence structure of a lifted 
$\mathrm{H}_{2} / \mathrm{N}_{2}$ jet flame in a vitiated co-flow, Flow Turbul. Combust. 76 (2006) 61-81.

[46] H. Wang, S.B. Pope, Lagrangian investigation of local extinction, re-ignition and auto-ignition in turbulent flames, Combust. Theor. Model. 12 (2008) 857-882.

[47] Z. Ren, S.B. Pope, Sensitivity calculations in PDF modelling of turbulent flames, Proc. Combust. Inst. 32 (2009) 1629-1637.

[48] K. Gkagkas, R.P. Lindstedt, The impact of reduced chemistry on auto-ignition of $\mathrm{H}_{2}$ in turbulent flows, Combust. Theor. Model. 13 (2009) 607-643.

[49] J. Lee, Y. Kim, DQMOM based PDF transport modeling for turbulent lifted nitrogen-diluted hydrogen jet flame with autoignition, Int. J. Hydrogen Energy 37 (2012) 18498-18508.

[50] S.M. Najafizadeh, M.T Sadeghi, R. Sotudeh-Gharebagh, D. Roekaerts, Chemical structure of autoignition in a turbulent lifted $\mathrm{H}_{2} / \mathrm{N}_{2}$ jet flame issuing into a vitiated coflow, Combust. Flame 160 (2013) 2928-2940.

[51] S.M. Najafizadeh, M.T. Sadeghi, R. Sotudeh-Gharebagh, Analysis of autoignition of a turbulent lifted $\mathrm{H}_{2} / \mathrm{N}_{2}$ jet flame issuing into a vitiated coflow, Int. J. Hydrogen Energy 38 (2013) 2510-2522.

[52] S.S. Patwardhan, S. De, K.N. Lakshmisha, B.N. Raghunandan, CMC simulations of lifted turbulent jet flame in a vitiated coflow, Proc. Combust. Inst. 32 (2009) 1705-1712.

[53] C. Duwig, L. Fuchs, Large eddy simulation of a $\mathrm{H}_{2} / \mathrm{N}_{2}$ lifted flame in a vitiated co-flow, Combust. Sci. Technol. 180 (2008) 453-480.

[54] S. Navarro-Martinez, A. Kronenburg, Flame stabilization mechanisms in lifted flames, Flow Turbul. Combust. 87 (2011) 377-406.

[55] I. Stanković, B. Merci, LES-CMC simulations of a turbulent lifted hydrogen flame in vitiated co-flow, Therm. Sci. 17 (2013) 763-772.

[56] K. Luo, H. Wang, F. Yi, J. Fan, Direct numerical simulation study of an experimental lifted $\mathrm{H}_{2} / \mathrm{N}_{2}$ flame. Part 1: validation and flame structure, Energ. Fuel 26 (2012) 6118-6127.

[57] S.B. Pope, Computationally efficient implementation of combustion chemistry using in situ adaptive tabulation, Combust. Theor. Model. 1 (1997) 41-63.

[58] C.D. Pierce, P. Moin, Progress-variable approach for large-eddy simulation of non-premixed turbulent combustion, J. Fluid Mech. 504 (2004) 73-97.

[59] P. Moin, K. Squires, W. Cabot, S. Lee, A dynamic subgrid-scale model for compressible turbulence and scalar transport, Phys. Fluids 3 (1991) 2746-2757.

[60] O. Desjardins, V. Moureau, H. Pitsch, High order conservative finite difference scheme for variable density low mach number turbulent flows, J. Comput. Phys. 227 (2008) 7125-7159.

[61] C.D. Pierce, Progress-variable approach for large-eddy simulation of turbulent combustion, Ph.D. thesis, Stanford University (2001).

[62] V. Raman, H. Pitsch, A consistent LES/filtered-density function formulation for the simulation of turbulent flames with detailed chemistry, Proc. Combust. Inst. 31 (2007) 1711-1719.

[63] P.J. Colucci, F.A. Jaberi, P. Givi, S.B. Pope, Filtered density function for large eddy simulation of turbulent reacting flows, Phys. Fluids 10 (2) (1998) 499.

[64] F.A. Jaberi, P.J. Colucci, S. James, P. Givi, S.B. Pope, Filtered mass density function for large-eddy simulation of turbulent reacting flows, J. Fluid Mech. 401 (1999) 85-121.

[65] J. Villermaux, L. Falk, A generalized mixing model for initial contacting of reactive fluids, Chem. Eng. Sci. 49 (1994) 5127-5140.

[66] R.J. Kee, G. Dixon-Lewis, J. Warnatz, M.E. Coltrin, J.A. Miller, A fortran computer code package for the evaluation of gas-phase multicomponent transport properties, Tech. Rep. SAND86-8246, Sandia National Laboratories, 1986.

[67] H. Wang, S.B. Pope, Large eddy simulation/probability density function modeling of a turbulent jet flame, Proc. Combust. Inst. 33 (2011) 1319-1330.

[68] M. Muradoglu, P. Jenny, S.B. Pope, D.A. Caughey, A consistent hybrid finite-volume/particle method for the PDF equations of turbulent reactive flows, J. Comput. Phys. 154 (1999) 342-371.

[69] M. Herrmann, G. Blanquart, V. Raman, Flux corrected finite volume scheme for preserving scalar boundedness in reacting large-eddy simulations, AIAA J. 44 (2006) 2879-2886. 
[70] J. Li, Z. Zhao, A. Kazakov, F.L. Dryer, An updated comprehensive kinetic model of hydrogen combustion, Int. J. Chem. Kinet. 36 (2004) 566-575.

[71] P.P. Popov, H. Wang, S.B. Pope, Specific volume coupling and convergence properties in hybrid particle/finite volume algorithms for turbulent reactive flows, J. Comput. Phys. 294 (2015) 110-126.

[72] E. Mastorakos, Ignition of turbulent non-premixed flames, Prog. Energy Combust. Sci. 35 (2009) 57-97.

[73] R.J. Kee, F.M. Rupley, E. Meeks, J.A. Miller, CHEMKIN-III: A fortran chemical kinetic package for the analysis of gas-phase chemical and plasma kinetics, Tech. Rep. SAND96-8216, Sandia National Laboratories, 1996.

[74] C.M. Kaul, V. Raman, G. Balarac, H. Pitsch, Numerical errors in the computation of subfilter scalar variance in large eddy simulations, Phys. Fluids 21 (2009) 055102.

[75] C.M. Kaul, V. Raman, A posteriori analysis of numerical errors in subfilter scalar variance modeling for large eddy simulation, Phys. Fluids 23 (2011) 035102.

[76] C.M. Kaul, V. Raman, Analysis of a dynamic model for subfilter scalar dissipation rate in large eddy simulation based on the subfilter scalar variance transport equation, Combust. Theor. Model. 17 (2013) 804-834. 


\section{List of Tables}

1 Jet flame boundary conditions. . . . . . . . . . . . . . . . . . . 22

2 Specification of the LES configurations. . . . . . . . . . . . . . . . . . 22 
Table 1: Jet flame boundary conditions.

\begin{tabular}{lccc}
\hline Parameter & Units & Jet & Coflow \\
\hline$d$ & $\mathrm{~m}$ & $4.57 \times 10^{-3}\left(=D_{\text {jet }}\right)$ & 0.21 \\
$U$ & $\mathrm{~m} / \mathrm{s}$ & $107\left(=U_{\text {jet }}\right)$ & 3.5 \\
$T$ & $\mathrm{~K}$ & 305 & 1045 \\
$X_{\mathrm{H}_{2}}$ & - & 0.25 & - \\
$X_{\mathrm{O}_{2}}$ & - & - & 0.1474 \\
$X_{\mathrm{N}_{2}}$ & - & 0.75 & 0.7534 \\
$X_{\mathrm{H}_{2} \mathrm{O}}$ & - & - & 0.0989 \\
\hline
\end{tabular}

Table 2: Specification of the LES configurations.

\begin{tabular}{ccc}
\hline Case & Differential diffusion & Dynamic mixing time-scale \\
\hline 1 & $\times$ & $\times$ \\
2 & $\times$ & $\sqrt{ }$ \\
3 & $\sqrt{ }$ & $\sqrt{ }$ \\
4 & $\sqrt{ }\left(D_{\mathrm{H}}=D_{\mathrm{H}_{2}}=0\right)$ & $\sqrt{ }$ \\
\hline
\end{tabular}




\section{List of Figures}

1 The burner geometry of Cabra et al. [10] for the lifted flame with vitiated coflow. . . . . . . . . . . . . . . . . . . .

2 Three snapshots (Case 3) of resolved temperature (top) and mass fraction of $\mathrm{OH}$ (bottom) showing the evolution of the flame towards a statistically stationary state. . . . . . . . . . . . . . . .

3 Radial profiles of time-averaged species diffusivities $\left(D_{\mathrm{H}}, D_{\mathrm{H}_{2}}, D_{\mathrm{OH}}, D_{\mathrm{O}_{2}}\right)$, turbulent diffusivity $D_{t}$, and mixture thermal diffusivity $\alpha$ at the different axial locations for Case $3 . \ldots \ldots \ldots \ldots$

4 Comparison of the instantaneous resolved temperature and mass fraction of $\mathrm{OH}$ without differential diffusion (Case 2) and with differential diffusion (Case $3)$. The inner isoline corresponds to the stoichiometric mixture fraction $\left(Z_{s t}=\right.$ $0.474)$ while the outer isoline shows the most reactive mixture fraction $\left(Z_{m r}=\right.$

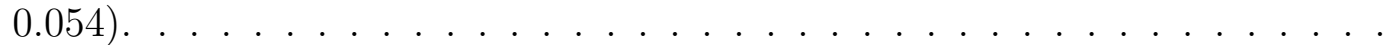

$5 \quad$ Radial profiles of mean mixture fraction and temperature at different axial locations for Cases 1 and $2 . \ldots \ldots \ldots \ldots$

6 Radial profiles of mean mass fractions of $\mathrm{H}_{2}$ and $\mathrm{OH}$ at different axial locations for Cases 1 and 2. . . . . . . . . . . . . . . . .

7 Radial profiles of mean mixture fraction at different axial locations for Cases

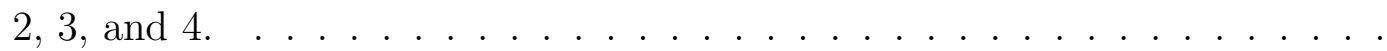

8 Radial profiles of RMS fluctuations of mixture fraction at different axial locations for Cases 2, 3, and 4. . . . . . . . . . . . . . . 29

9 Radial profiles of mean temperature at different axial locations for Cases 2,

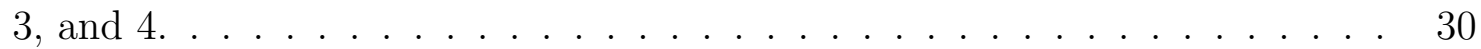

10 Radial profiles of RMS fluctuations of temperature at different axial locations for Cases 2, 3, and 4. . . . . . . . . . . . . . . . . 30

11 Radial profiles of mean $Y_{\mathrm{H}_{2}}$ at different axial locations for Cases 2, 3, and $4 . \quad 31$

12 Radial profiles of RMS fluctuations of $Y_{\mathrm{H}_{2}}$ at different axial locations for Cases

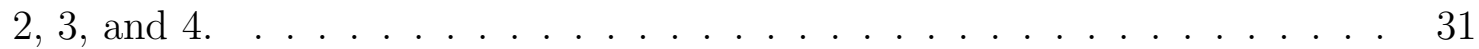

13 Radial profiles of mean $Y_{\mathrm{O}_{2}}$ at different axial locations for Cases 2, 3, and $4 . \quad 32$

14 Radial profiles of RMS fluctuations of $Y_{\mathrm{O}_{2}}$ at different axial locations for Cases

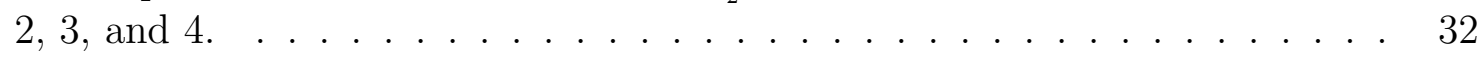

15 Radial profiles of mean $Y_{\mathrm{OH}}$ at different axial locations for Cases 2, 3, and 4.33

16 Radial profiles of RMS fluctuations of $Y_{\mathrm{OH}}$ at different axial locations for Cases 2, 3, and 4. . . . . . . . . . . . . . . . . . . . . . . 33

17 Radial profiles of mean $Y_{\mathrm{H}_{2} \mathrm{O}}$ at different axial locations for Cases 2, 3, and $4 . \quad 34$

18 Radial profiles of RMS fluctuations of $Y_{\mathrm{H}_{2} \mathrm{O}}$ at different axial locations for Cases 2, 3, and 4. . . . . . . . . . . . . . . . . . . . 34

19 Radial profiles of mean $Y_{\mathrm{N}_{2}}$ at different axial locations for Cases 2, 3, and $4 . \quad 35$

20 Radial profiles of RMS fluctuations of $Y_{\mathrm{N}_{2}}$ at different axial locations for Cases

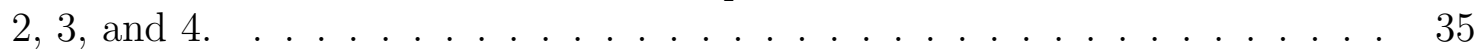

21 Conditional mean of temperature near the flame base. . . . . . . . . . . . 36 
22 Conditional mean of $Y_{\mathrm{OH}}$ near the flame base.

23 Measured and predicted lift-off height as a function of coflow temperature. Measurements of Cabra et al. [10] (diamond); Wu et al. [45] (open circle); Gordon et al. [26] (open triangle); Predictions of Cao et al. [23] (pentagram); Patwardhan et al. [52] (symbol X); Stankovic et al. [55] (hexagram); Naud et al. [15] (solid square); present study Cases 2 (solid triangle) and 3 (solid circle)

D.24 Radial profiles of mean density at different axial locations for Case 3. solid lines: LES calculations; dashed lines: PDF calculations. . . . . . . . . . . .

D.25 Time-averaged radial profiles mixture fraction (top), temperature (middle), and mass fraction of $\mathrm{OH}$ (bottom) computed using three different grids for

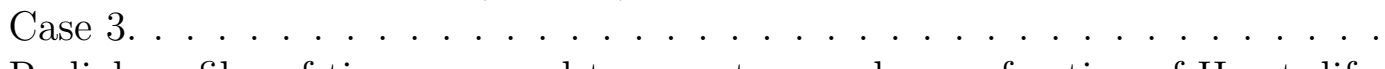

D.26 Radial profiles of time-averaged temperature and mass fraction of $\mathrm{H}_{2}$ at different axial locations for Case $1 \ldots \ldots \ldots \ldots$

D.27 Radial profiles of time-averaged $C_{Z}^{-1}$ (which is dynamically-determined $C_{\phi}$ )

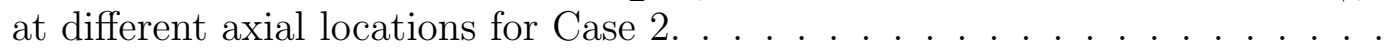

D.28 Radial profiles of mean and RMS fluctuations of mixture fraction, temperature, and mass fractions of $\mathrm{H}_{2}$ and $\mathrm{OH}$ at $x / D_{\text {jet }}=26$ for Cases 2, 3 and

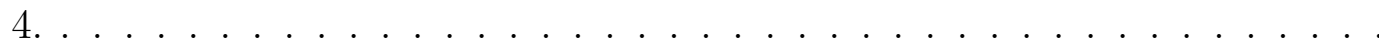




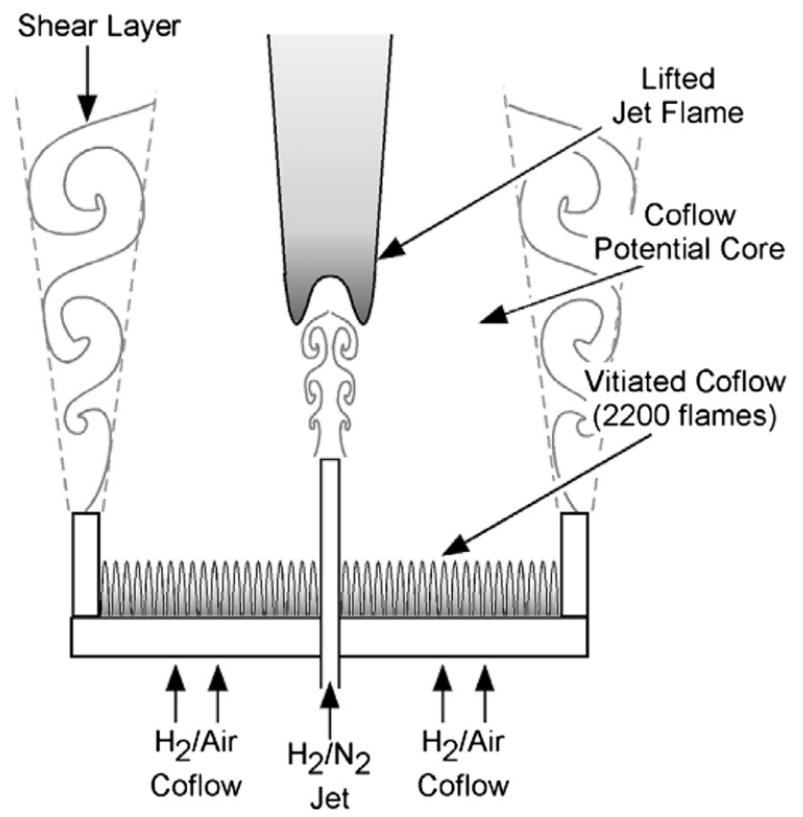

Figure 1: The burner geometry of Cabra et al. [10] for the lifted flame with vitiated coflow. 


\section{$\mathbf{T}[\mathrm{K}]$}

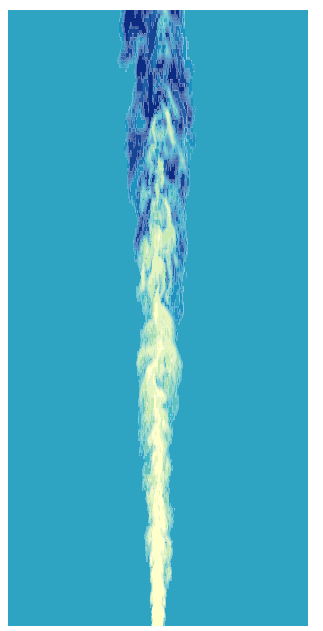

400600800100012001400

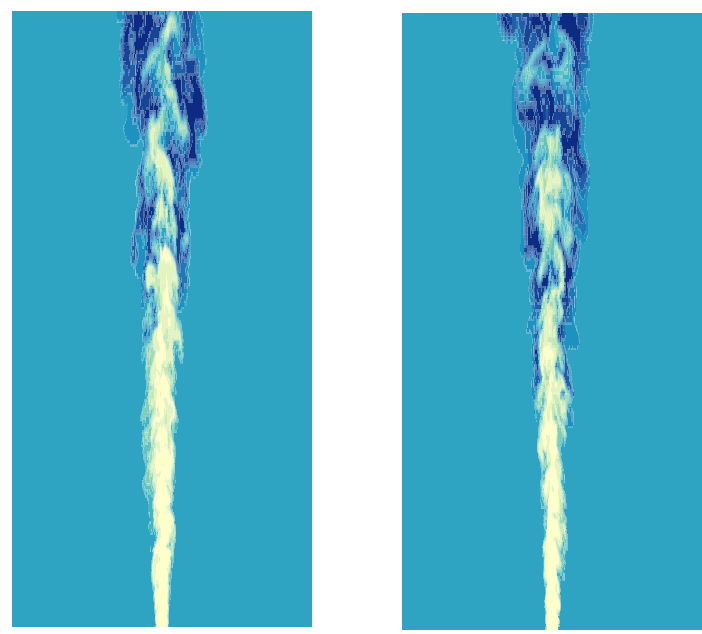

$\begin{array}{lllll}Y_{\mathrm{OH}} & & & & \\ & 0.0002 & 0.0008 & 0.0014 & 0.002\end{array}$
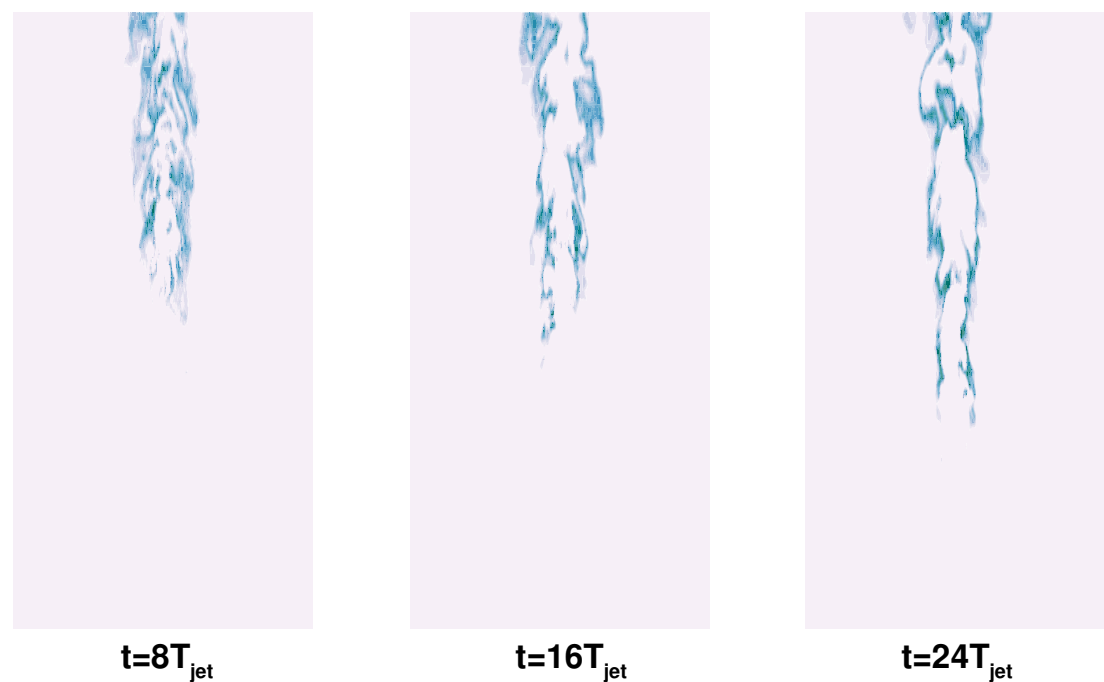

Figure 2: Three snapshots (Case 3) of resolved temperature (top) and mass fraction of OH (bottom) showing the evolution of the flame towards a statistically stationary state. 

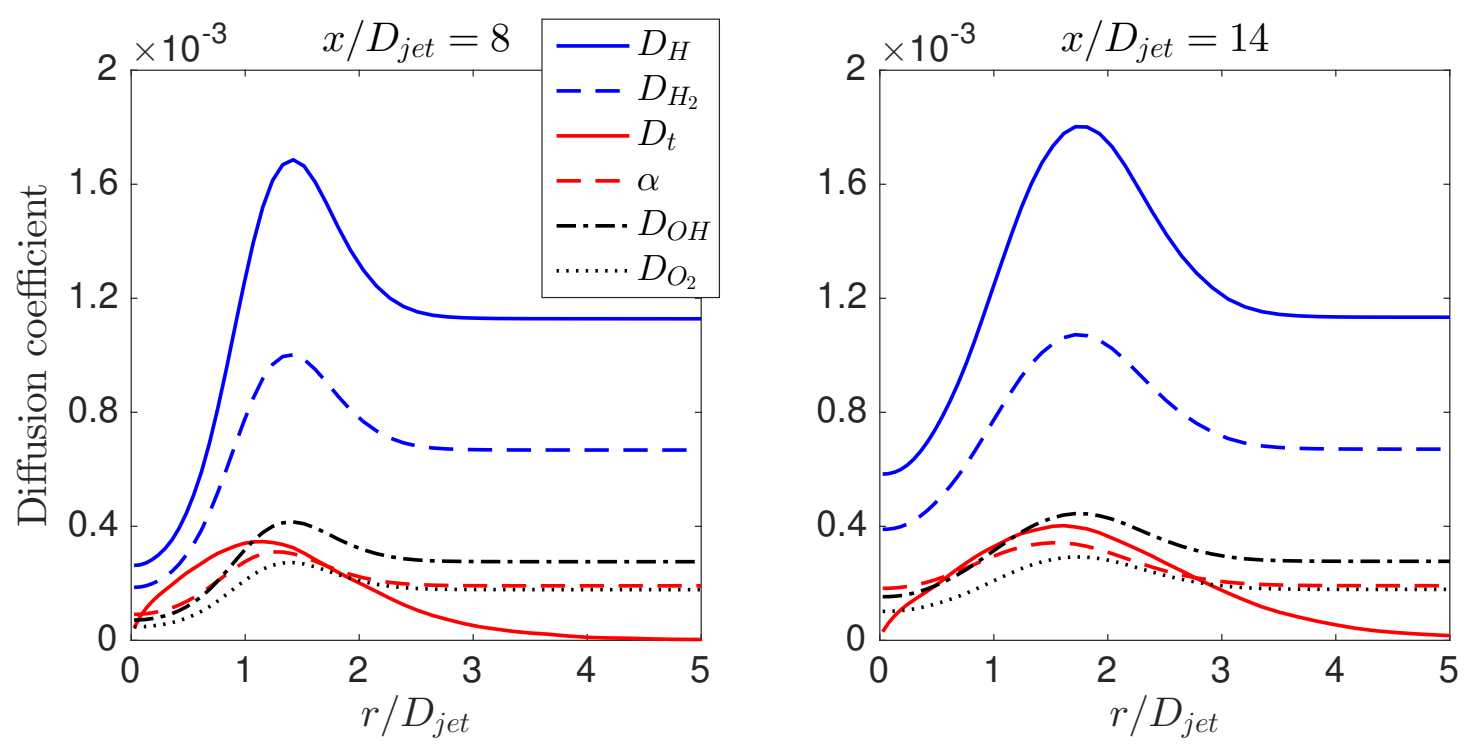

Figure 3: Radial profiles of time-averaged species diffusivities $\left(D_{\mathrm{H}}, D_{\mathrm{H}_{2}}, D_{\mathrm{OH}}, D_{\mathrm{O}_{2}}\right)$, turbulent diffusivity $D_{t}$, and mixture thermal diffusivity $\alpha$ at the different axial locations for Case 3.
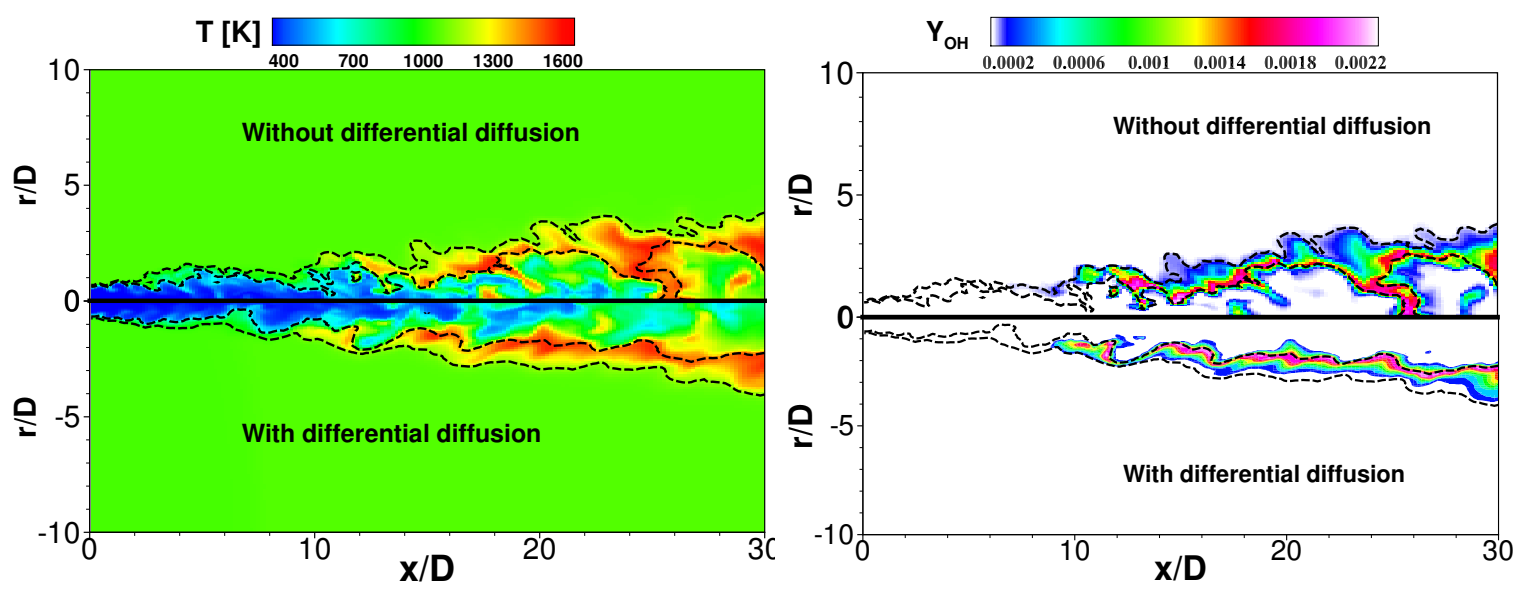

Figure 4: Comparison of the instantaneous resolved temperature and mass fraction of $\mathrm{OH}$ without differential diffusion (Case 2) and with differential diffusion (Case 3). The inner isoline corresponds to the stoichiometric mixture fraction $\left(Z_{s t}=0.474\right)$ while the outer isoline shows the most reactive mixture fraction $\left(Z_{m r}=\right.$ $0.054)$. 

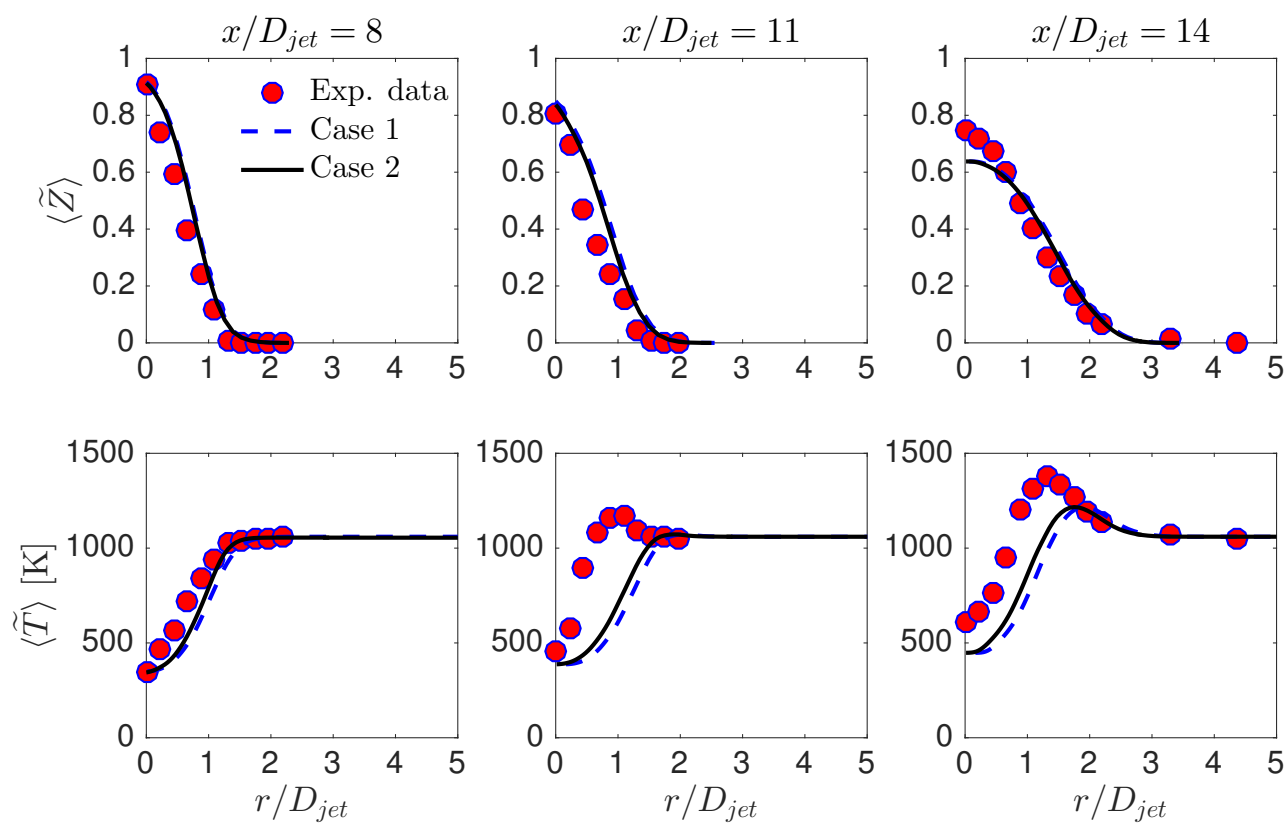

Figure 5: Radial profiles of mean mixture fraction and temperature at different axial locations for Cases 1 and 2 .
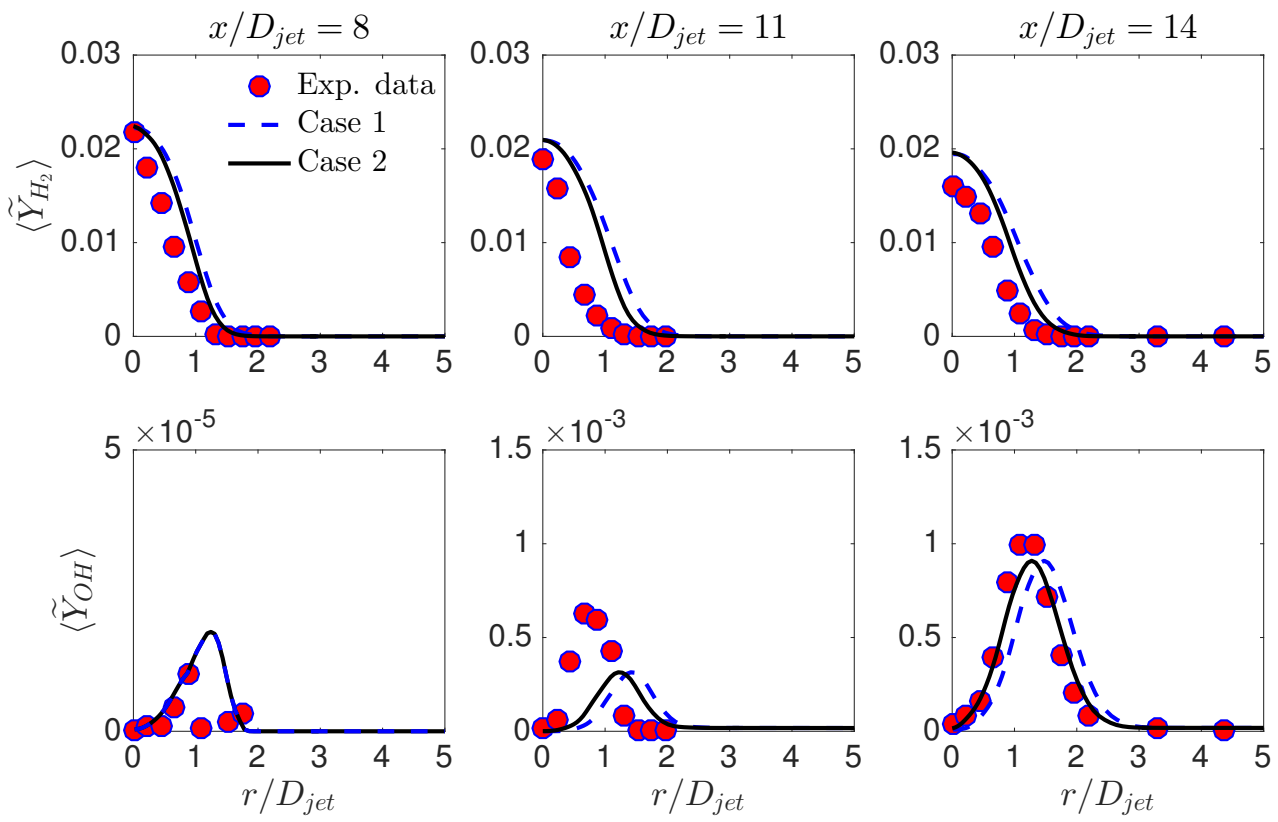

Figure 6: Radial profiles of mean mass fractions of $\mathrm{H}_{2}$ and $\mathrm{OH}$ at different axial locations for Cases 1 and 2. 

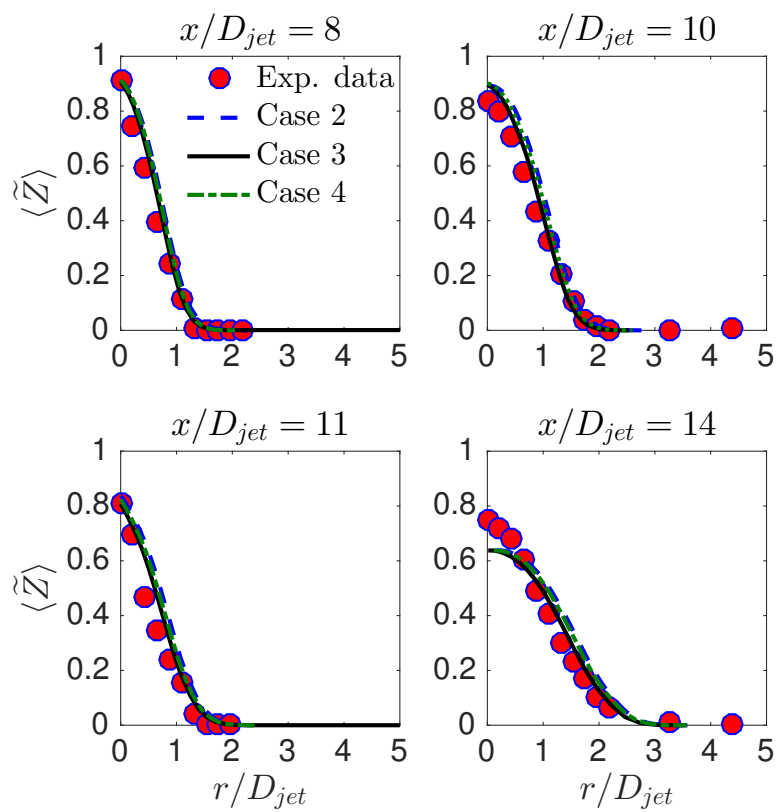

Figure 7: Radial profiles of mean mixture fraction at different axial locations for Cases 2, 3, and 4 .
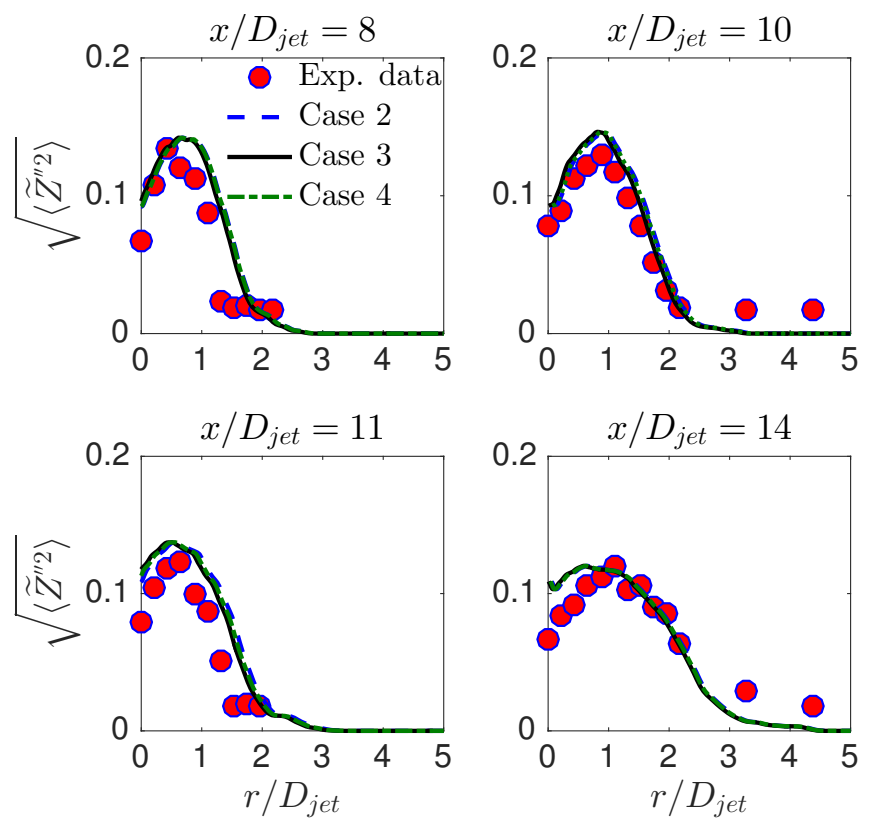

Figure 8: Radial profiles of RMS fluctuations of mixture fraction at different axial locations for Cases 2, 3, and 4. 

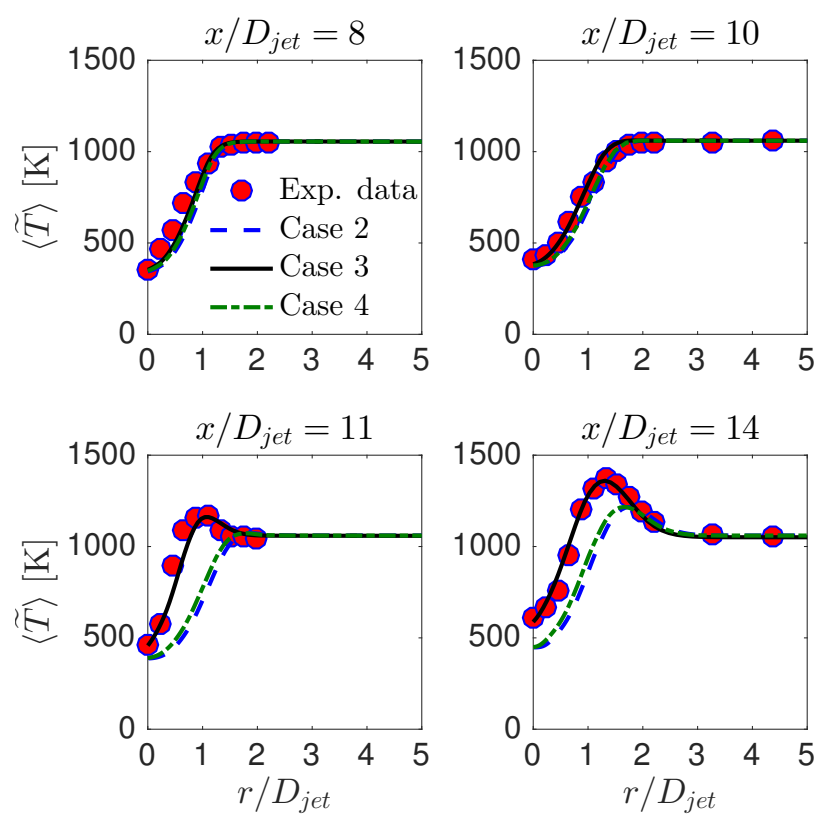

Figure 9: Radial profiles of mean temperature at different axial locations for Cases 2, 3, and 4.
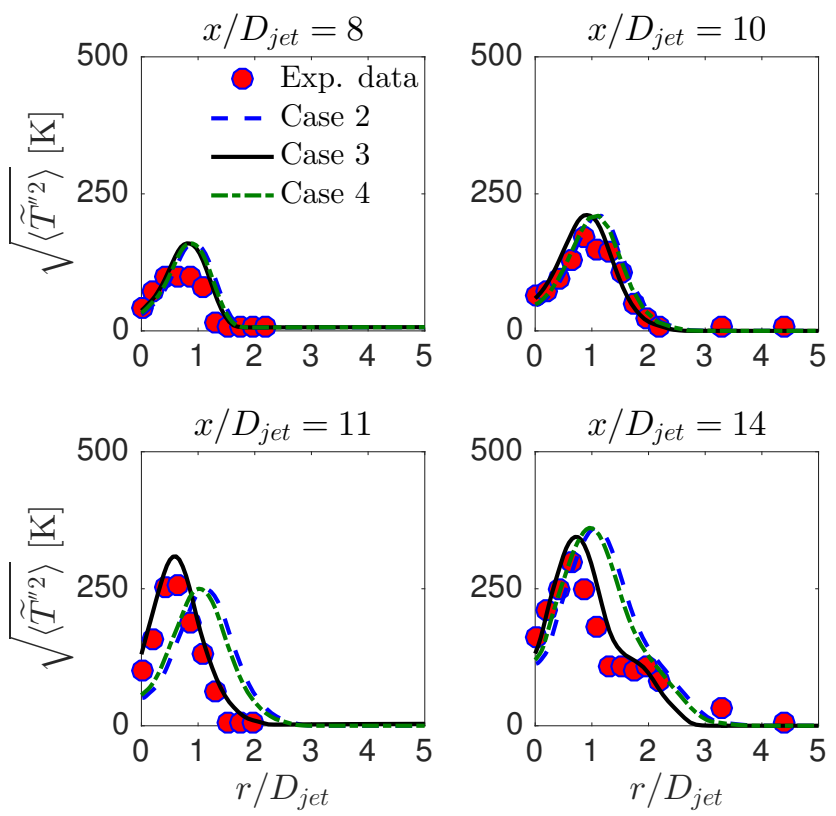

Figure 10: Radial profiles of RMS fluctuations of temperature at different axial locations for Cases 2,3 , and 4 . 

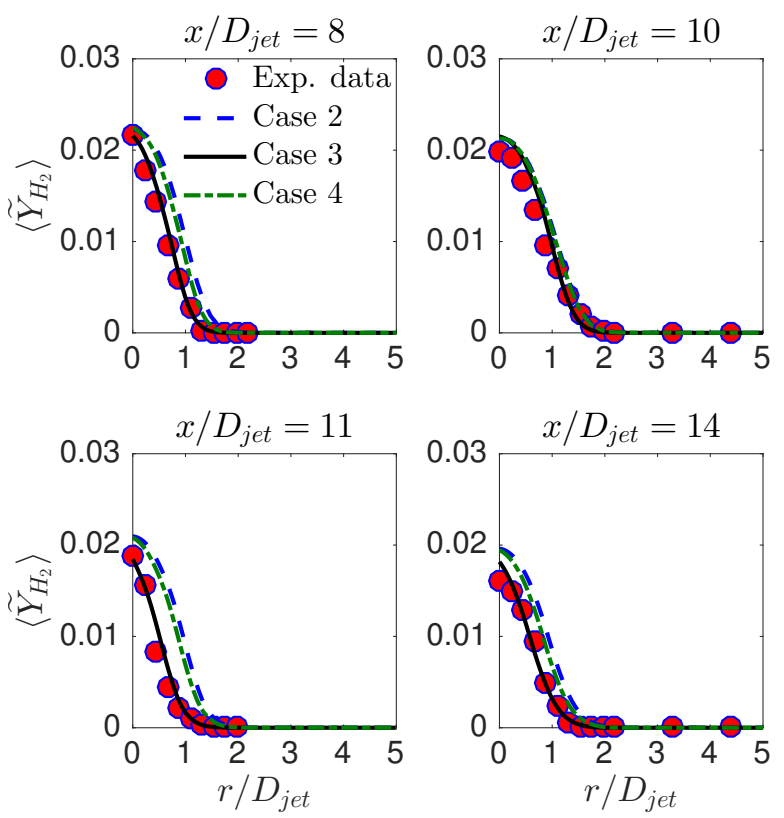

Figure 11: Radial profiles of mean $Y_{\mathrm{H}_{2}}$ at different axial locations for Cases 2, 3, and 4 .
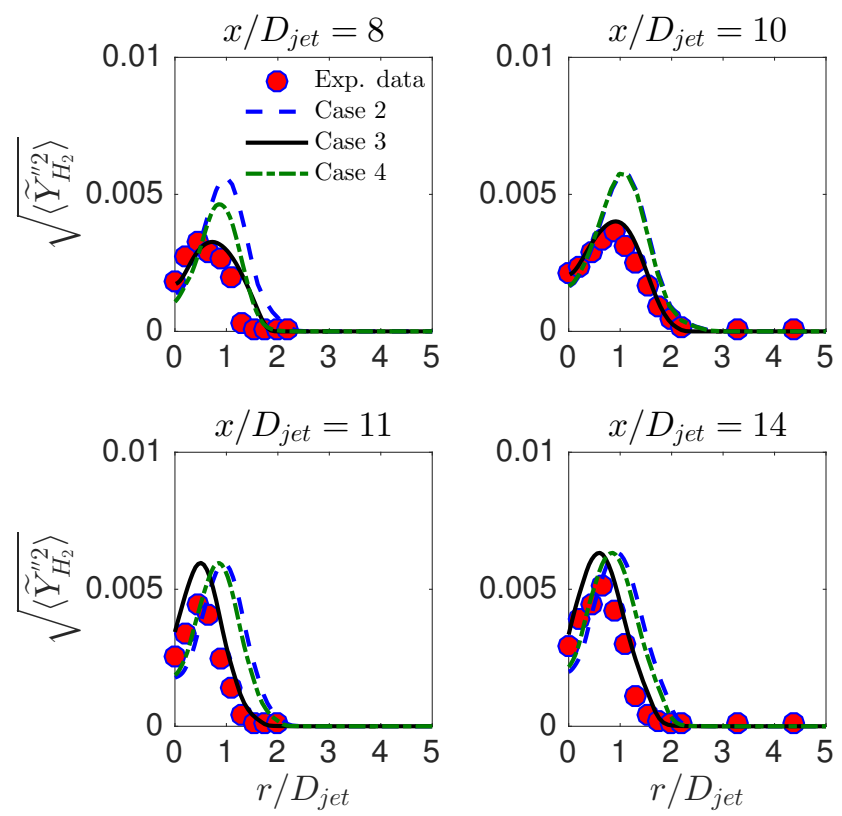

Figure 12: Radial profiles of RMS fluctuations of $Y_{\mathrm{H}_{2}}$ at different axial locations for Cases 2, 3, and 4 . 

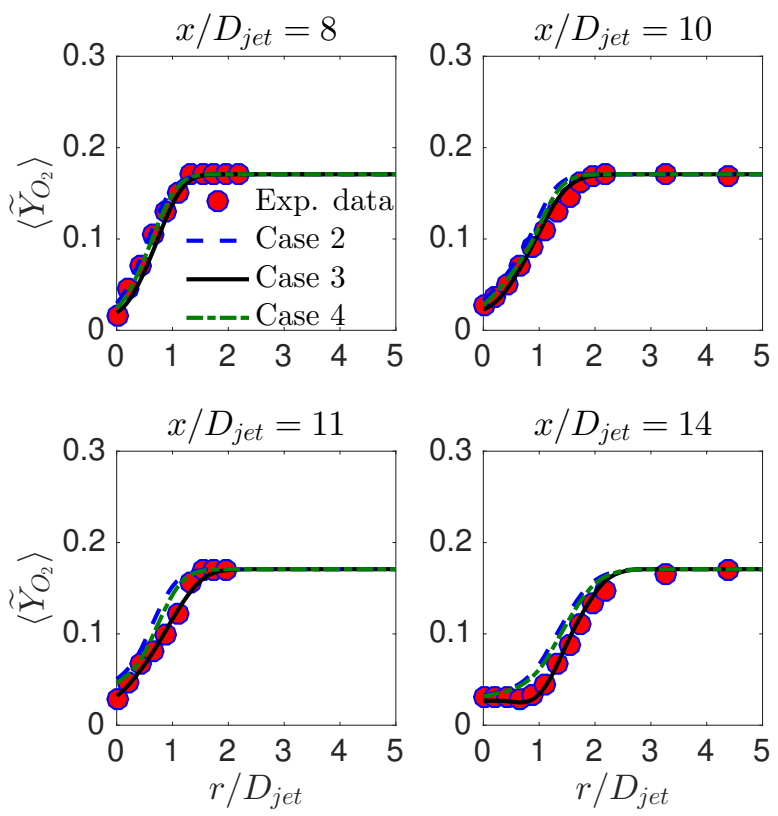

Figure 13: Radial profiles of mean $Y_{\mathrm{O}_{2}}$ at different axial locations for Cases 2, 3, and 4 .
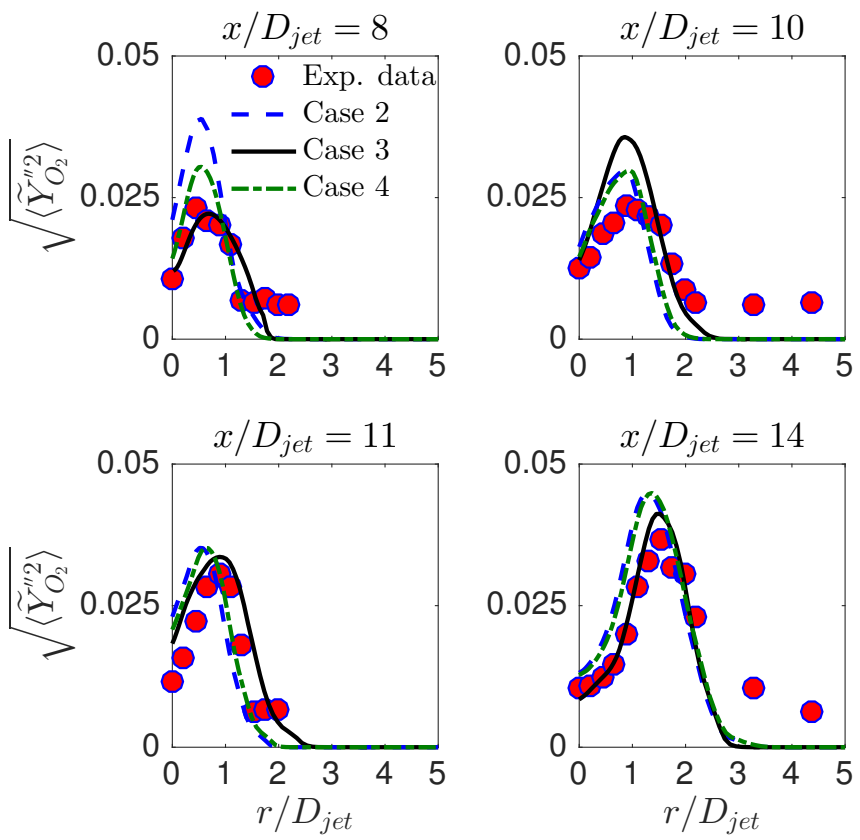

Figure 14: Radial profiles of RMS fluctuations of $Y_{\mathrm{O}_{2}}$ at different axial locations for Cases 2, 3, and 4 . 

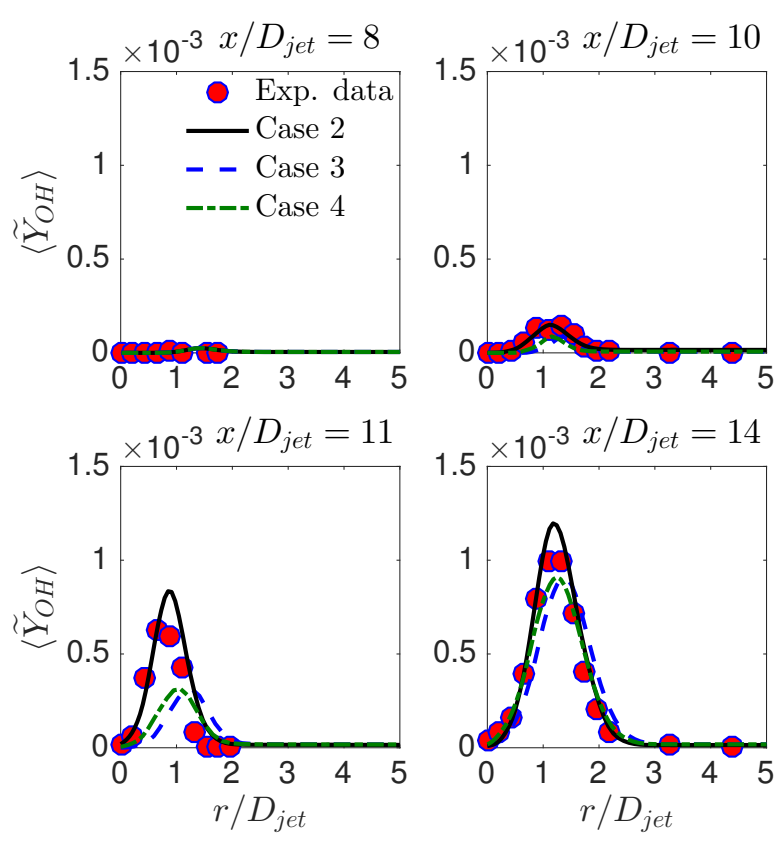

Figure 15: Radial profiles of mean $Y_{\mathrm{OH}}$ at different axial locations for Cases 2, 3, and 4.
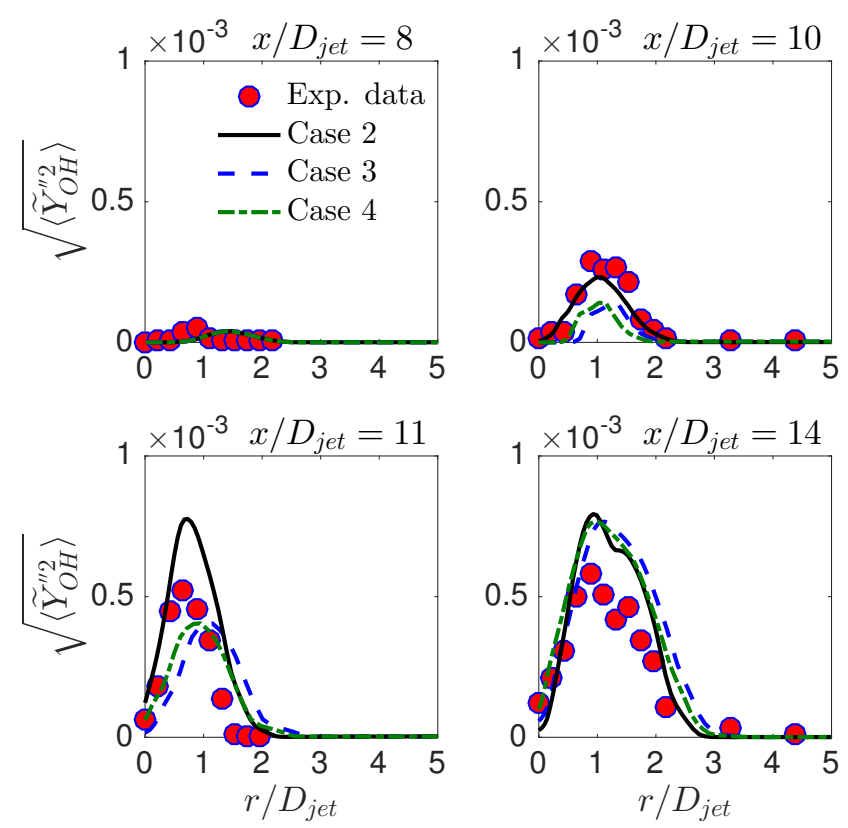

Figure 16: Radial profiles of RMS fluctuations of $Y_{\mathrm{OH}}$ at different axial locations for Cases 2, 3, and 4. 

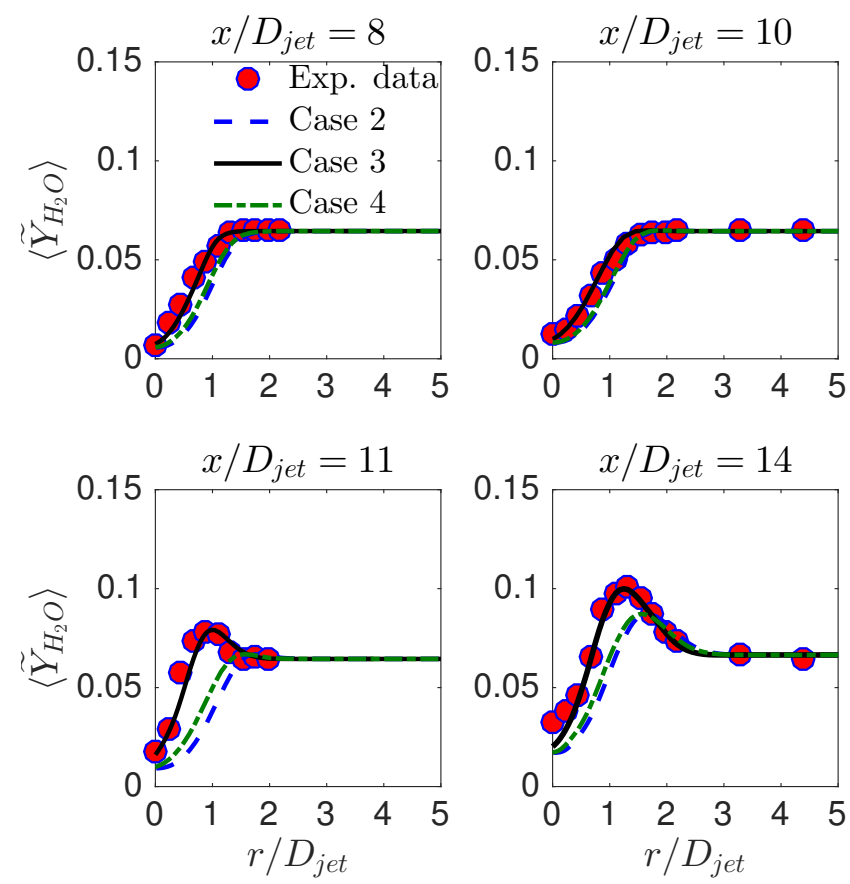

Figure 17: Radial profiles of mean $Y_{\mathrm{H}_{2} \mathrm{O}}$ at different axial locations for Cases 2, 3, and 4 .
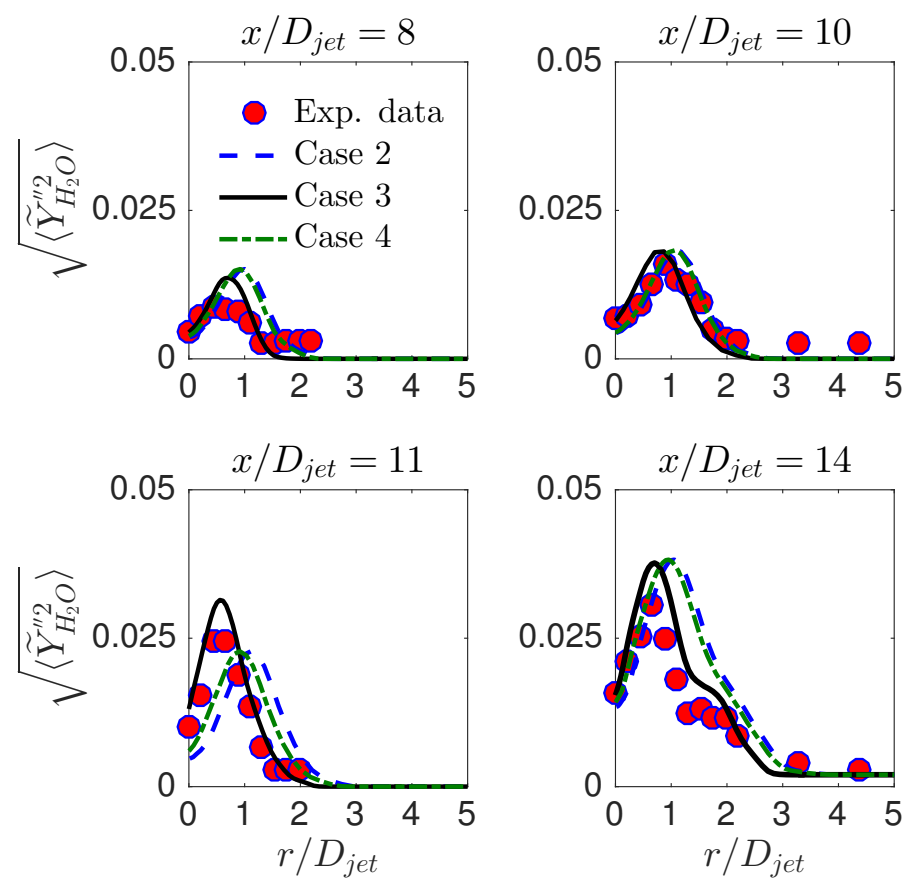

Figure 18: Radial profiles of RMS fluctuations of $Y_{\mathrm{H}_{2} \mathrm{O}}$ at different axial locations for Cases 2, 3, and 4. 

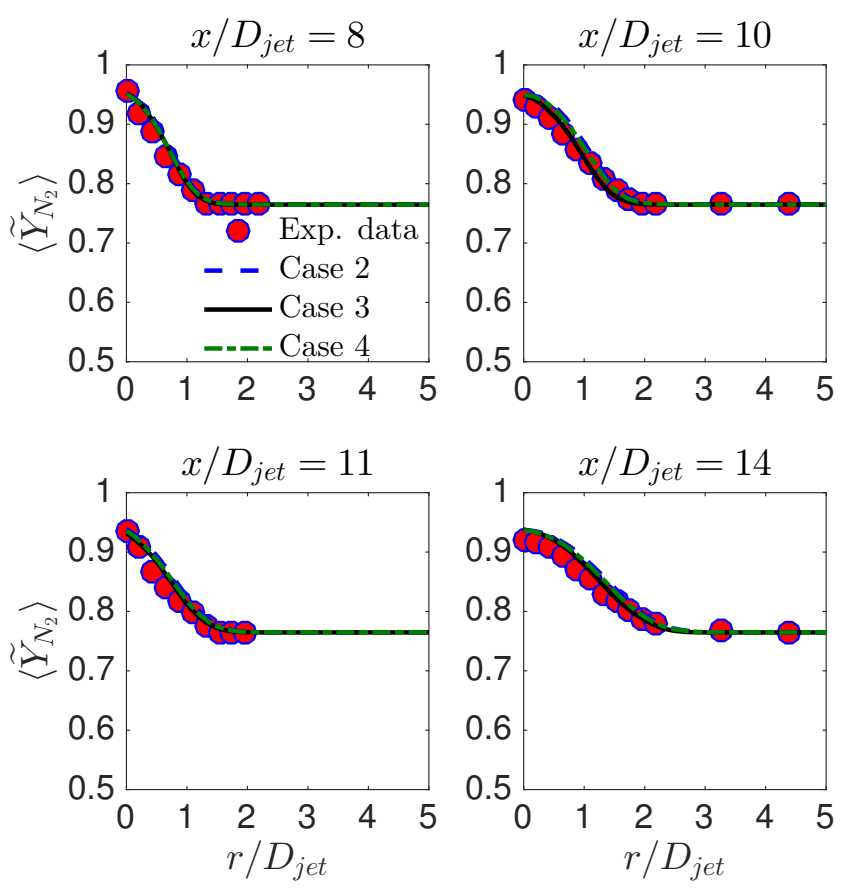

Figure 19: Radial profiles of mean $Y_{\mathrm{N}_{2}}$ at different axial locations for Cases 2, 3, and 4 .
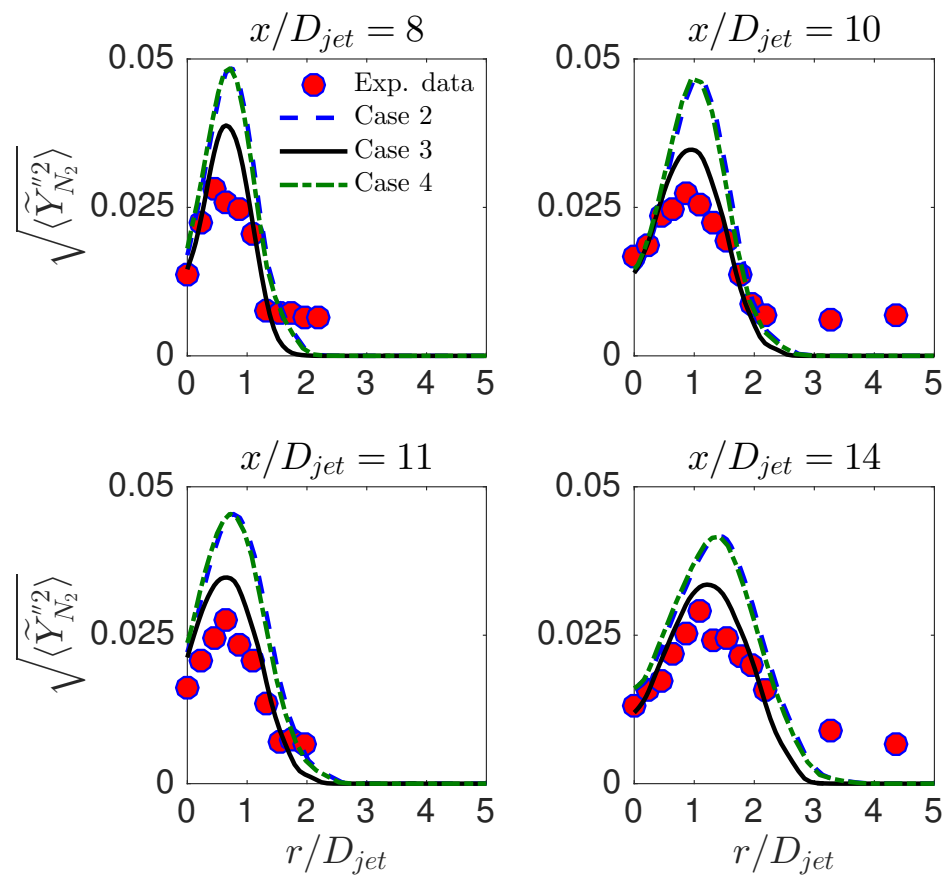

Figure 20: Radial profiles of RMS fluctuations of $Y_{\mathrm{N}_{2}}$ at different axial locations for Cases 2, 3, and 4 . 


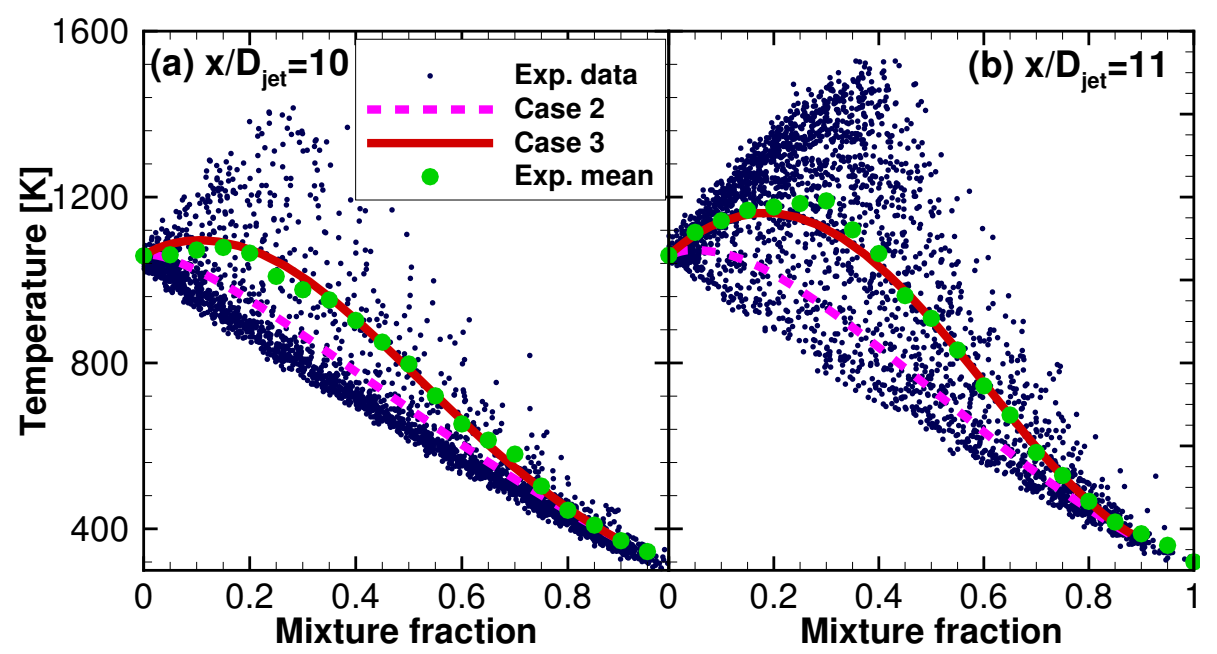

Figure 21: Conditional mean of temperature near the flame base.

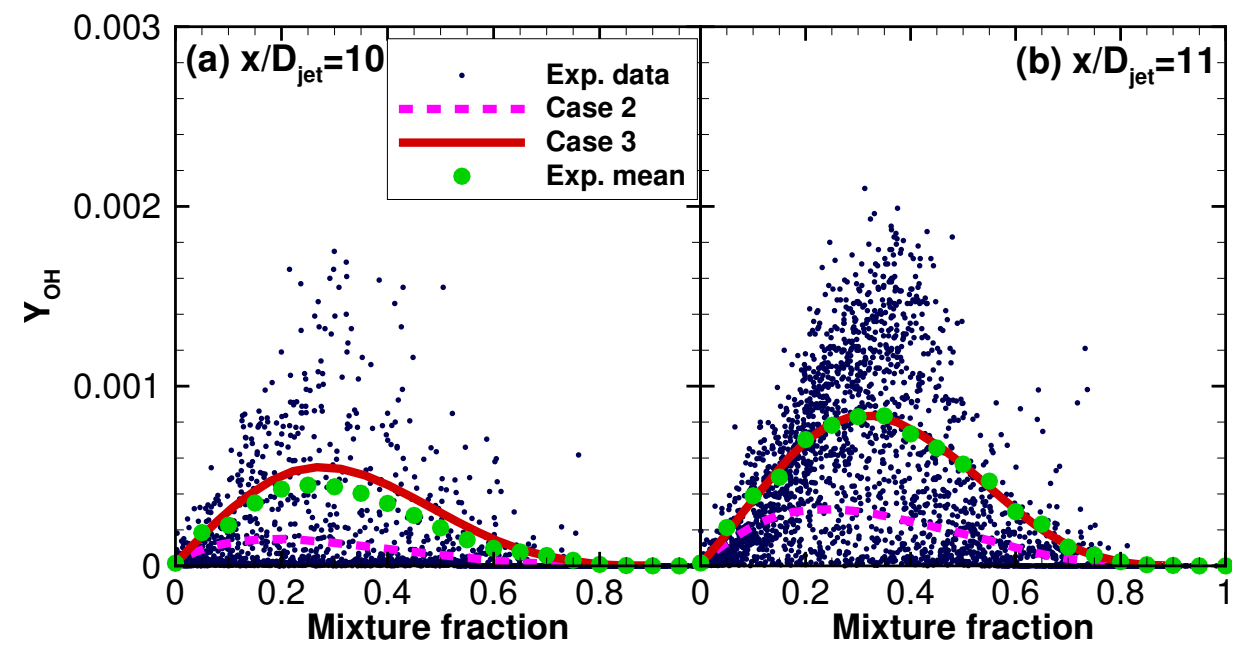

Figure 22: Conditional mean of $Y_{\mathrm{OH}}$ near the flame base. 


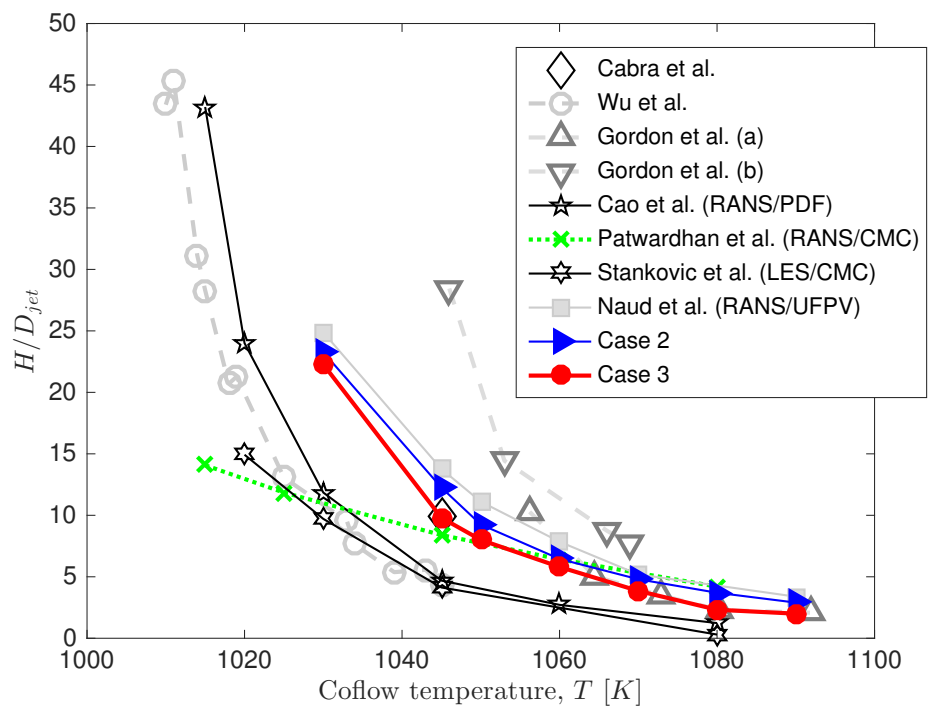

Figure 23: Measured and predicted lift-off height as a function of coflow temperature. Measurements of Cabra et al. [10] (diamond); Wu et al. [45] (open circle); Gordon et al. [26] (open triangle); Predictions of Cao et al. [23] (pentagram); Patwardhan et al. [52] (symbol X); Stankovic et al. [55] (hexagram); Naud et al. [15] (solid square); present study Cases 2 (solid triangle) and 3 (solid circle). 


\section{Appendix A. Consistency between LES and PDF mean density}

Unlike the coupling algorithm proposed by Popov et al. [71], there is no relaxation term in the present scalar transport equation that is used to calculate density. The consistency between LES and PDF mean fields obtained by using present coupling algorithm has been validated in $[43,62]$. To verify the performance of the present coupling algorithm, radial profiles of mean density at different axial locations for Case 3 are plotted in Fig. D.24. It is seen that the density calculations from LES and PDF are in good agreement. The slight discrepancy is due to the different numerical errors involved in the LES and PDF implementations. The numerical consistency of the density from LES and PDF makes the present two-way coupling algorithm acceptable for the particular lifted flame.

\section{Appendix B. Effect of grid resolution}

In order to understand the role of spatial resolution on these results, the effect of filter width on the profiles is presented here. Note that a change in grid spacing effectively alters the relative importance of sub-filter and resolved scales. More importantly, at relatively coarse scales, the influence of numerical errors increases, leading to a corruption of the resolved fields [74-76]. Further, strong velocity and scalar gradients are inadequately resolved. Hence, a minimum grid resolution is needed to ensure that such numerical effects do not dominate the simulation results. Figure D.25 shows the time-averaged statistics at four axial locations $x / D=8,10,11$, and 14 for Case 3 .

Three sets of grids are considered in the simulations, of sizes $N_{x} \times N_{r} \times N_{\theta}=120 \times 72 \times 32$, $192 \times 108 \times 32$, and $320 \times 180 \times 32$ in axial, radial, and azimuthal directions, respectively. It is seen that the results from the baseline $(192 \times 108 \times 32)$ and finest $(320 \times 180 \times 32)$ grids are in good agreement with experimental data, especially for scalar fields. In contrast, the results from the coarse grid $(120 \times 72 \times 32)$ are significantly different from the experimental results. This comparison demonstrates that the results obtained with the baseline grid are accurate enough (numerically) to analyze the effect of differential diffusion.

\section{Appendix C. Effect of $C_{\phi}$}

To evaluate the sensitivity of the predictions to the values of $C_{\phi}$, the radial profiles of time-averaged temperature and mass fraction of $\mathrm{H}_{2}$ at different axial locations for Case 1 are shown in Fig. D.26. It is seen that the value of the mixing constant $C_{\phi}$ has a relatively small effect on the predictions. For the particular flame that is largely chemically controlled, the computed results obtained using different values of $C_{\phi}$ are almost identical. There is minimal change once $C_{\phi} \geq 10$. We use this as the choice for optimal value for the coefficient.

To assess the effect of dynamically-determined $C_{\phi}$ (i.e., $C_{Z}^{-1}$ ), we first examine the radial profiles of time-averaged $C_{Z}^{-1}$ at different axial locations for Case 2 in Fig. D.27. It is seen that the value of $C_{Z}^{-1}$ deviates evidently from 2 which is typically adopted in the modeling of conditional mixing term in Eq. (7). Figure D.27 shows that in the near field at $x / D_{\text {jet }}=1$, the variation of the value of $C_{Z}^{-1}$ with radial location is evident inside the mixing layer between central jet and coflow stream. At progressive downstream locations 
$\left(x / D_{\text {jet }}=1,8,14,26\right)$, the value of $C_{Z}^{-1}$ becomes closer to the optimal value of 10 inside the mixing layer, and it tends to 20 outside of the mixing layer. $C_{Z}^{-1}$ reaches the maximum value in the laminar flow region outside of the mixing layer. It is expected that using the dynamically-determined $C_{\phi}$ can not only remove the need to specify the value of model constant but also more accurately and cost-effectively represent the mixing time-scales of reactive scalars. Nevertheless, Figs. 5 and 6 show that accounting for the dynamic mixing time-scale model only slightly improves computed results in the present study. This can be attributed to the observation that large values of $C_{Z}^{-1}$ are located outside the mixing layer where the RMS mixture fraction is zero.

\section{Appendix D. Results at $x / D_{j e t}=26$}

Radial profiles of time-averaged and RMS mixture fraction, temperature, and mass fractions of $\mathrm{H}_{2}$ and $\mathrm{OH}$ at $x / D_{\text {jet }}=26$ are illustrated in Fig. D.28. It is seen that the computed and measured mean and RMS profiles are in good overall agreement for Case 3. Similar to results at $x / D_{\text {jet }}=8,10,11,14$, the mixture fraction profiles are insensitive to the differential diffusion, since in the present study we assume that the mixture fraction is a conserved scalar and that the source term due to differential diffusion in the transport equation of mixture fraction is neglected. The mean temperature and mass fraction of $\mathrm{OH}$ for Case 2 are underpredicted in the mixing-region between central jet and coflow stream. This underprediction is due to that fact that differential diffusion cannot be adequately presented by the conventional IEM model for Case 2. On the other side, Case 3 yields considerably improved predictions, especially in the shear layer between coflow and jet stream. Similar to Case 2, the mean temperature of Case 4 is also underpredicted, which demonstrates that $\mathrm{H}$ and $\mathrm{H}_{2}$ are two critical radicals that play an important role in differential diffusion effect. The higher mean values of $\mathrm{H}_{2}$ predicted for Case 2 are consistent with the discrepancies in the prediction of mean temperature. While mean predictions are in very good agreement with experimental data, slight overpredicitions of RMS temperature and $\mathrm{H}_{2}$ are observed. Since the grid used here is stretched in the axial direction, the filter width increases with downstream distance. This slightly degrades the accuracy of the models as well as the numerical schemes.

The above comparison analysis shows that the effect of differential diffusion is not negligible and that accounting for molecular transport can significantly improve the predictions of mixture fraction, temperature, and species mass fractions. Moreover, it is found that $\mathrm{H}$ and $\mathrm{H}_{2}$ are responsible for the differences in predictions of Cases 3 and 4 . 

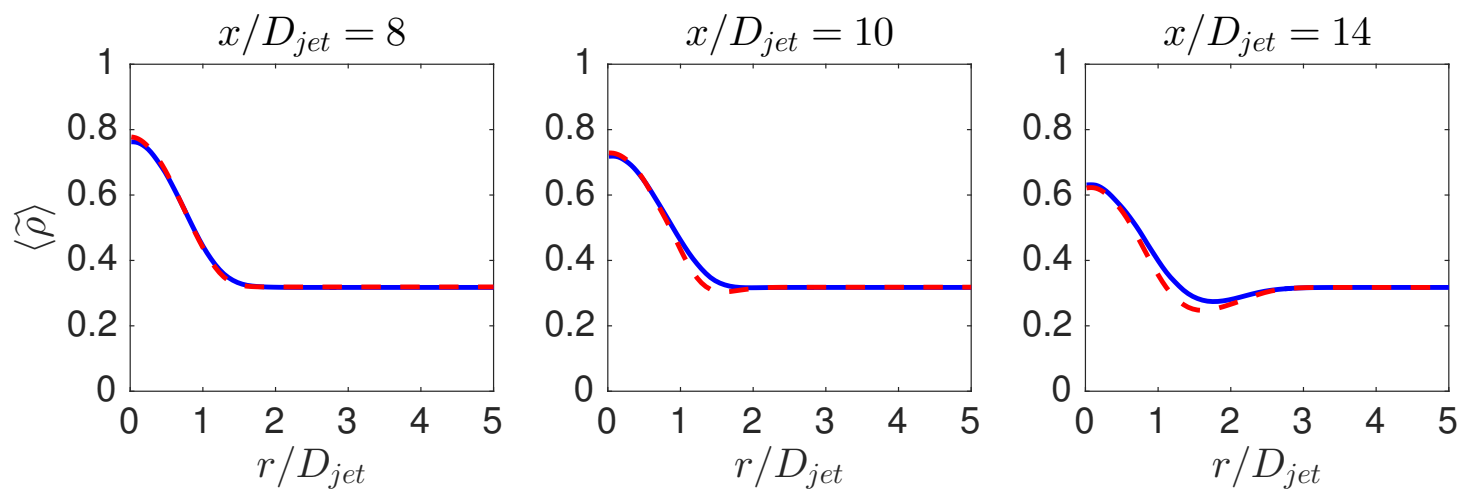

Figure D.24: Radial profiles of mean density at different axial locations for Case 3. solid lines: LES calculations; dashed lines: PDF calculations.

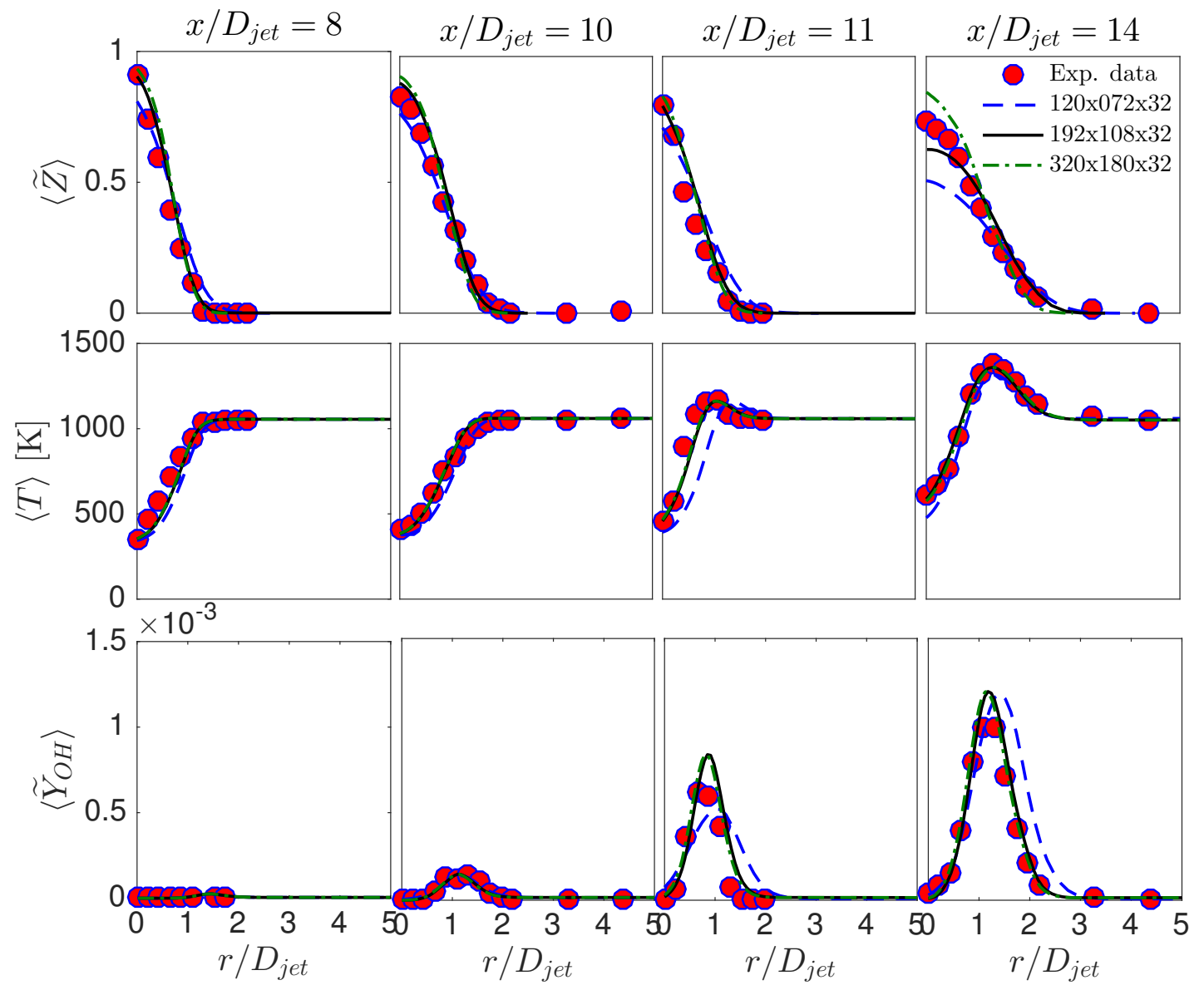

Figure D.25: Time-averaged radial profiles mixture fraction (top), temperature (middle), and mass fraction of $\mathrm{OH}$ (bottom) computed using three different grids for Case 3. 

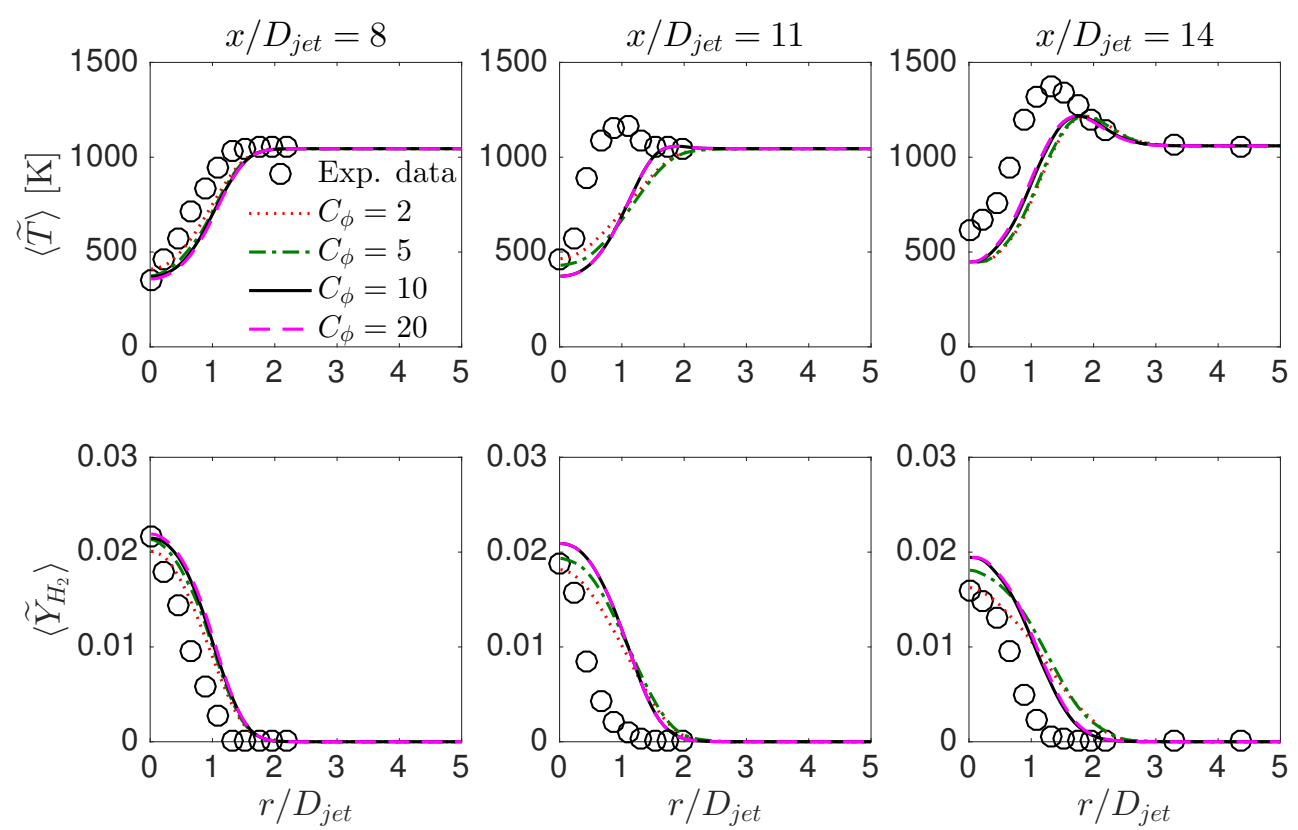

Figure D.26: Radial profiles of time-averaged temperature and mass fraction of $\mathrm{H}_{2}$ at different axial locations for Case 1. 

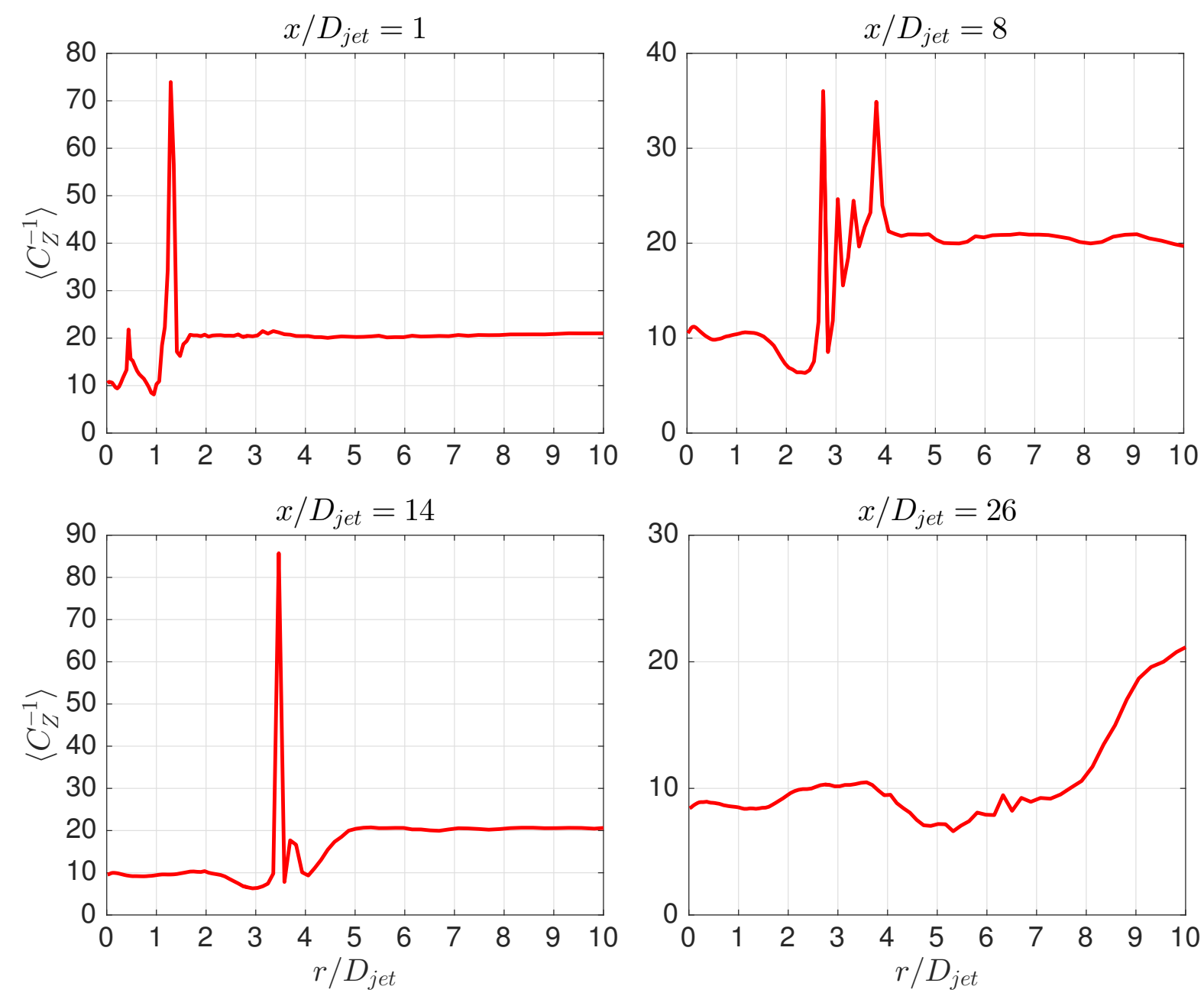

Figure D.27: Radial profiles of time-averaged $C_{Z}^{-1}$ (which is dynamically-determined $C_{\phi}$ ) at different axial locations for Case 2 . 

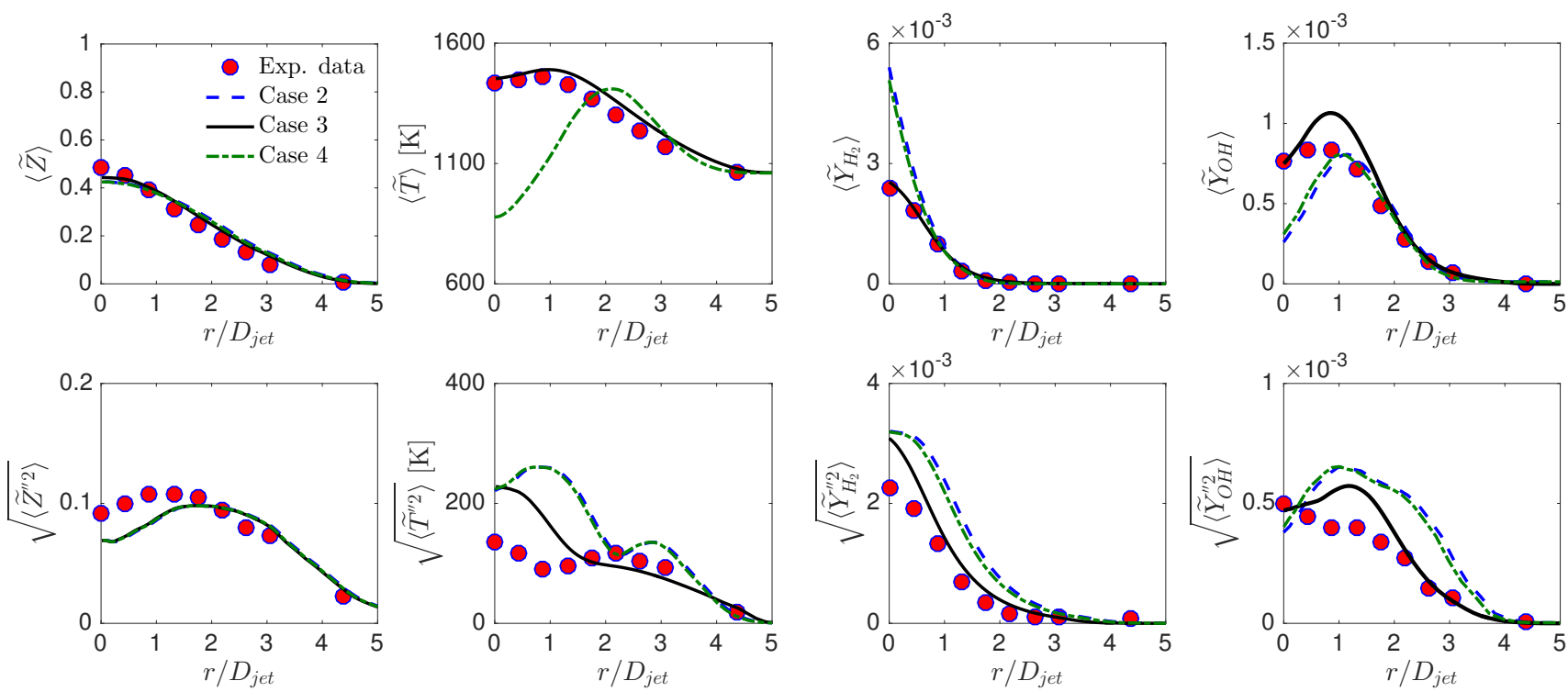

Figure D.28: Radial profiles of mean and RMS fluctuations of mixture fraction, temperature, and mass fractions of $\mathrm{H}_{2}$ and $\mathrm{OH}$ at $x / D_{j e t}=26$ for Cases 2,3 and 4 . 